Article

\title{
Genetic Potential of the Biocontrol Agent Pseudomonas brassicacearum (Formerly P. trivialis) 3Re2-7 Unraveled by Genome Sequencing and Mining, Comparative Genomics and Transcriptomics
}

\author{
Johanna Nelkner ${ }^{1}{ }^{\circledR}$, Gonzalo Torres Tejerizo ${ }^{2}$, Julia Hassa ${ }^{1}$, Timo Wentong Lin ${ }^{1}$, Julian Witte ${ }^{1}$, \\ Bart Verwaaijen ${ }^{1}$, Anika Winkler ${ }^{1}$, Boyke Bunk ${ }^{3}$, Cathrin Spröer ${ }^{3}$, Jörg Overmann ${ }^{3}$, \\ Rita Grosch ${ }^{4} \mathbb{D}$, Alfred Pühler ${ }^{1}$ and and Andreas Schlüter ${ }^{1, * \mathbb{D}}$ \\ 1 Center for Biotechnology (CeBiTec), Bielefeld University, Genome Research of Industrial Microorganisms, \\ Universitätsstraße 27, 33615 Bielefeld, Germany \\ 2 Facultad de Ciencias Exactas, Departamento de Ciencias Biologicas, IBBM, \\ Universidad Nacional de La Plata, Calle 115 y 47, 1900 La Plata, Argentina \\ 3 Leibniz-Institute DSMZ-German Collection of Microorganisms and Cell Cultures, Inhoffenstraße 7B, \\ 38124 Braunschweig, Germany \\ 4 Leibniz-Institute of Vegetable and Ornamental Crops (IGZ), Plant-Microbe Systems, \\ Theodor-Echtermeyer-Weg 1, 14979 Großbeeren, Germany \\ * Correspondence: aschluet@CeBiTec.Uni-Bielefeld.DE; Tel.: +49-(0)521-106-8757
}

Received: 11 July 2019; Accepted: 6 August 2019; Published: 9 August 2019

\begin{abstract}
The genus Pseudomonas comprises many known plant-associated microbes with plant growth promotion and disease suppression properties. Genome-based studies allow the prediction of the underlying mechanisms using genome mining tools and the analysis of the genes unique for a strain by implementing comparative genomics. Here, we provide the genome sequence of the strain Pseudomonas brassicacearum 3Re2-7, formerly known as $P$. trivialis and P. reactans, elucidate its revised taxonomic classification, experimentally verify the gene predictions by transcriptome sequencing, describe its genetic biocontrol potential and contextualize it to other known Pseudomonas biocontrol agents. The P. brassicacearum 3Re2-7 genome comprises a circular chromosome with a size of 6,738,544 bp and a GC-content of $60.83 \%$. 6267 genes were annotated, of which 6113 were shown to be transcribed in rich medium and/or in the presence of Rhizoctonia solani. Genome mining identified genes related to biocontrol traits such as secondary metabolite and siderophore biosynthesis, plant growth promotion, inorganic phosphate solubilization, biosynthesis of lipo- and exopolysaccharides, exoproteases, volatiles and detoxification. Core genome analysis revealed, that the 3Re2-7 genome exhibits a high collinearity with the representative genome for the species, P. brassicacearum subsp. brassicacearum NFM421. Comparative genomics allowed the identification of 105 specific genes and revealed gene clusters that might encode specialized biocontrol mechanisms of strain 3Re2-7. Moreover, we captured the transcriptome of P. brassicacearum 3Re2-7, confirming the transcription of the predicted biocontrol-related genes. The gene clusters coding for 2,4-diacetylphloroglucinol (phlABCDEFGH) and hydrogen cyanide ( $h c n A B C$ ) were shown to be highly transcribed. Further genes predicted to encode putative alginate production enzymes, a pyrroloquinoline quinone precursor peptide PqqA and a matrixin family metalloprotease were also found to be highly transcribed. With this study, we provide a basis to further characterize the mechanisms for biocontrol in Pseudomonas species, towards a sustainable and safe application of P. brassicacearum biocontrol agents.
\end{abstract}

Keywords: Pseudomonas brassicacearum; genome mining; RNA sequencing; comparative genomics; biocontrol; plant-growth promotion; transcriptomics 


\section{Introduction}

Productivity in agriculture has been increased, for example, by application of fertilizers and pesticides. However, there is a serious concern that land use intensification in agriculture results in large-scale ecosystem degradation, loss of crop productivity and reduction of biodiversity [1]. Plants are associated with a high number of microbes of which some are pathogenic while others feature plant beneficial properties. Plant diseases like bare patch of cereals, brown patch of turf, root canker of lucerne and black scurf of potato caused by plant pathogens as for example the fungus Rhizoctonia solani can lead to yield losses. It was estimated that such soilborne pathogens are responsible for up to $25 \%$ yield losses in relevant crops worldwide [2]. Effective strategies to control soilborne pathogens are limited today. In terms of disease control, it is well-documented that beneficial plant-associated microbes can protect plants from disease infestations and prevent the deficiencies caused by exclusive reliance on pesticides [3,4]. Biocontrol, the concept of using beneficial microbes for pathogen control as strategy for sustainable agriculture is established and has been studied intensively over the last years as an alternative for the use of chemical pesticides. Microbial biocontrol agents (BCAs) have been shown to be involved in direct suppression of pathogens [5], as well as in inducing systemic resistance in plants to enhance tolerance to different environmental stresses [6]. These biocontrol characteristics have been linked to their ability to produce compounds featuring antimicrobial activity, siderophores and chelators, exoproteases, phosphorus solubilization, as well as plant growth regulation, stimulation and signaling molecules $[7,8]$.

In a study addressing the effect of long-term farming practices, the genus Pseudomonas has been shown to be abundant in agricultural soils [9]. Many soilborne pseudomonads have the potential to suppress plant pathogens and promote plant growth and health, making them candidates as effective BCAs [3]. Especially the group of fluorescent Pseudomonas species is studied intensively and used as effective biocontrol agent against various plant pathogens [10]. Members of this group have great potential in biocontrol because of their ability to produce secondary metabolites relevant in suppression of pathogens [11-15].

The species Pseudomonas brassicacearum also comprises plant-associated strains obtained from the root surface (rhizoplane), the zone around the root (rhizosphere) as well as endophytes of different plant hosts [16-21]. Most known P. brassicacearum strains were described as potentially phyto-beneficial; further they have been shown to produce antimicrobial compounds and feature activity against phytopathogenic microbes [16-21]. The type strain of the species is P. brassicacearum DBK11 (CFBP $11706^{T} /$ DSM $13227^{T}$ ) [16] but only its $16 \mathrm{~S}$ rDNA sequence is available so far. Therefore the genome of P. brassicacearum subsp. brassicacearum NFM421 [18] was chosen as reference for genome-based studies regarding this species $[19,22]$.

Gene clusters such as the phlABCDEFGH and $h c n A B C$ operons responsible for the biosynthesis of 2,4-diacetylphloroglucinol (DAPG) and hydrogen cyanide $(\mathrm{HCN})$ were detected in the genomes of P. brassicacearum strains. DAPG and HCN have been linked to biocontrol traits [23]. Furthermore, genes involved in the production of 1-aminocyclopropane-1-carboxylate deaminase (acdS) and pyrroloquinoline-quinone ( $p q q A B C D E F)$ were predicted in genomes of $P$. brassicacearum strains and have been associated with plant growth-promotion (PGP). Also, different secretion systems, genes for the biosynthesis of different volatile compounds and exoproteases have been identified and are likely to contribute to the biocontrol potential of this species.

Subject of this study is the strain Pseudomonas brassicacearum 3Re2-7, formerly known as P. trivialis 3Re2-7 [24] and P. reactans 3Re2-7 [25]. It was originally isolated from the endorhiza of potato in a study addressing the screening of potential biocontrol agents in large scale [25]. The strain 3Re2-7 was selected as successful biocontrol strain able to suppress soilborne pathogens such as Rhizoctonia solani on lettuce and sugar beet [26]. Moreover, it was shown to be able to produce proteases and siderophores and to harbour the phlD gene which is involved in the production of DAPG. In a follow-up study involving 20 isolates which are antagonistic to R. solani, it was shown to be one of the two most effective strains and also featured a positive effect on plant growth in vitro. At that time, it was 
classified as Pseudomonas reactans, based on its 16S rDNA sequence and fatty acid methyl ester profile $[25,26]$. Further, being unlikely to cause diseases in humans, it was placed into the low risk group 1 (classification according to Technical Rules for Biological Agents (TRBA) from the German Federal Institute for Occupational Safety and Health, TRBA 466 "Classification of prokaryotes (bacteria and archaea) into risk groups"). In 2006, it was reclassified as $P$. trivialis. Moreover, it was shown to produce 12 different small volatile organic compounds (VOCs), most representing unidentified compounds but undecadiene, undecene and (benzyloxy)benzonitrile were identified [24]. Also, an antagonistic effect of these VOCs on R. solani has been shown in this study. In another study, a low and short term effect of strain 3Re2-7 on the indigenous plant-associated microbial community has been proved [27]. The strain 3Re2-7 is commercially available as growth enhancing crop inoculant.

Like most genome sequences nowadays, those of $P$. brassicacearum strains, have been published only in short formats (Short Genome Communications and Genome Announcements), lacking both a comprehensive genomic and a comparative study of the species [18-22].

In this study, we established the complete genome sequence of the BCA P. brassicacearum 3Re2-7. We aim to give an overview and classification of the genetic biocontrol determinants encoded in this biocontrol agent by mining its genome sequence. Using various bioinformatics tools and databases, we screen for known genes and features but also identify potentially new features by similarity, motif structures and cluster structures. By implementing comparative genomics of publicly available complete and draft genome sequences of $P$. brassicacearum species, we provide an overview of the P. brassicacearum taxon. We thereby focus on strain 3Re2-7 to elucidate its unique genetic potential. Further, we apply RNA-Seq to monitor the transcription of biocontrol genes. By combining bioinformatics approaches of genome mining, comparative genomics and transcriptomics, we aim to improve the understanding of biocontrol traits in P. brassicacearum species. The analyses provide the basis to further characterize the mechanisms for niche adaptation, antagonism towards phytopathogenic fungi, as well as plant growth promotion in P. brassicacearum.

\section{Results and Discussion}

\subsection{Genome Sequencing and General Genome Features of P. brassicacearum 3Re2-7}

In order to establish the genome sequence of the biocontrol strain P. brassicacearum 3Re2-7 and enable genome-based analyses, its genome was sequenced using a combination of PacBio and Illumina technologies. The obtained reads were assembled into one high-quality continuous contig of 6,738,544 bases, featuring a GC content of $60.8 \%$ and an average coverage of 104-fold. Figure 1 shows a circular representation of the 3Re2-7 chromosome. In total, 6267 genes, of which 5992 are annotated as protein-encoding sequences (CDSs), 190 pseudo genes, 65 tRNAs, 4 ncRNAs and 16 rRNA genes could be predicted. Four prophage regions were identified using PHASTER [28], of which three regions were predicted to be complete (P1, Synechococcus phage S-CAM7, NCBI accession NC_031927; P3, Pseudomonas phage YMC11/02/R656, NC_028657 and P4, Enterobacter phage Arya, NC_031048) and one region (P2, Pseudomonas phage phiPSA1, NC_024365) is incomplete. The localizations of these prophage regions are indicated in Figure 2.

No antimicrobial resistance genes could be detected using ResFinder-3.1 [29]. Moreover, neither virulence genes, resistance genes, pathogen-associated genes nor homologs of these were detected by means of IslandViewer 4 [30]. 


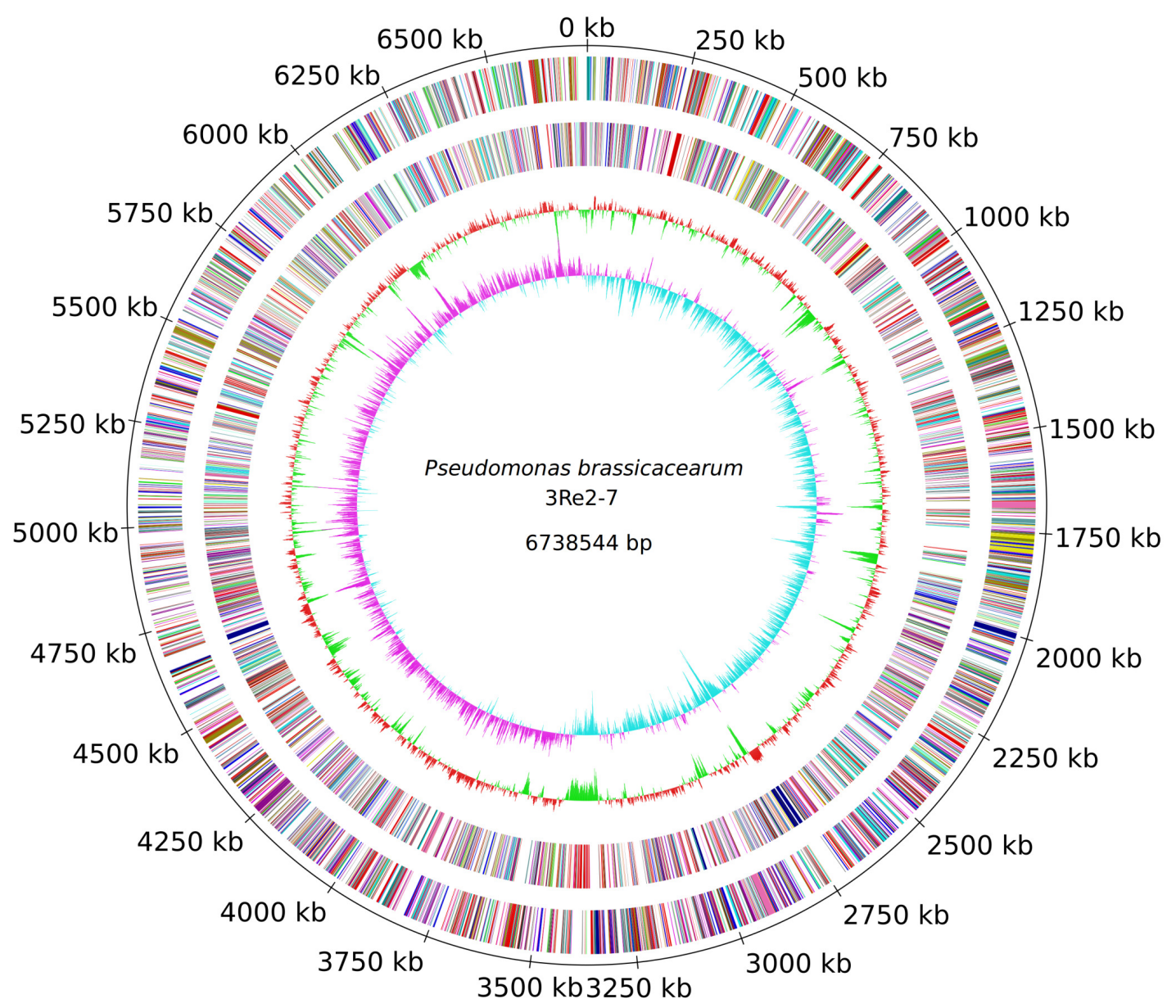

Figure 1. Circular representation of the P. brassicacearum 3Re2-7 chromosome. From the outer to the inner concentric circle: Circle 1, genomic position in kb (total 6,738,544 bp); the replication initiation gene $d n a A$ was selected as first gene of the circular chromosome. Circles 2 and 3, predicted protein-coding sequences (CDS) on the forward [transcribed clockwise] (outer part) and the reverse [transcribed counter clockwise] (inner part) strand colored according to the assigned COG classes. Circle 4, GC content showing deviations from the average. Circle 5, GC skew. 


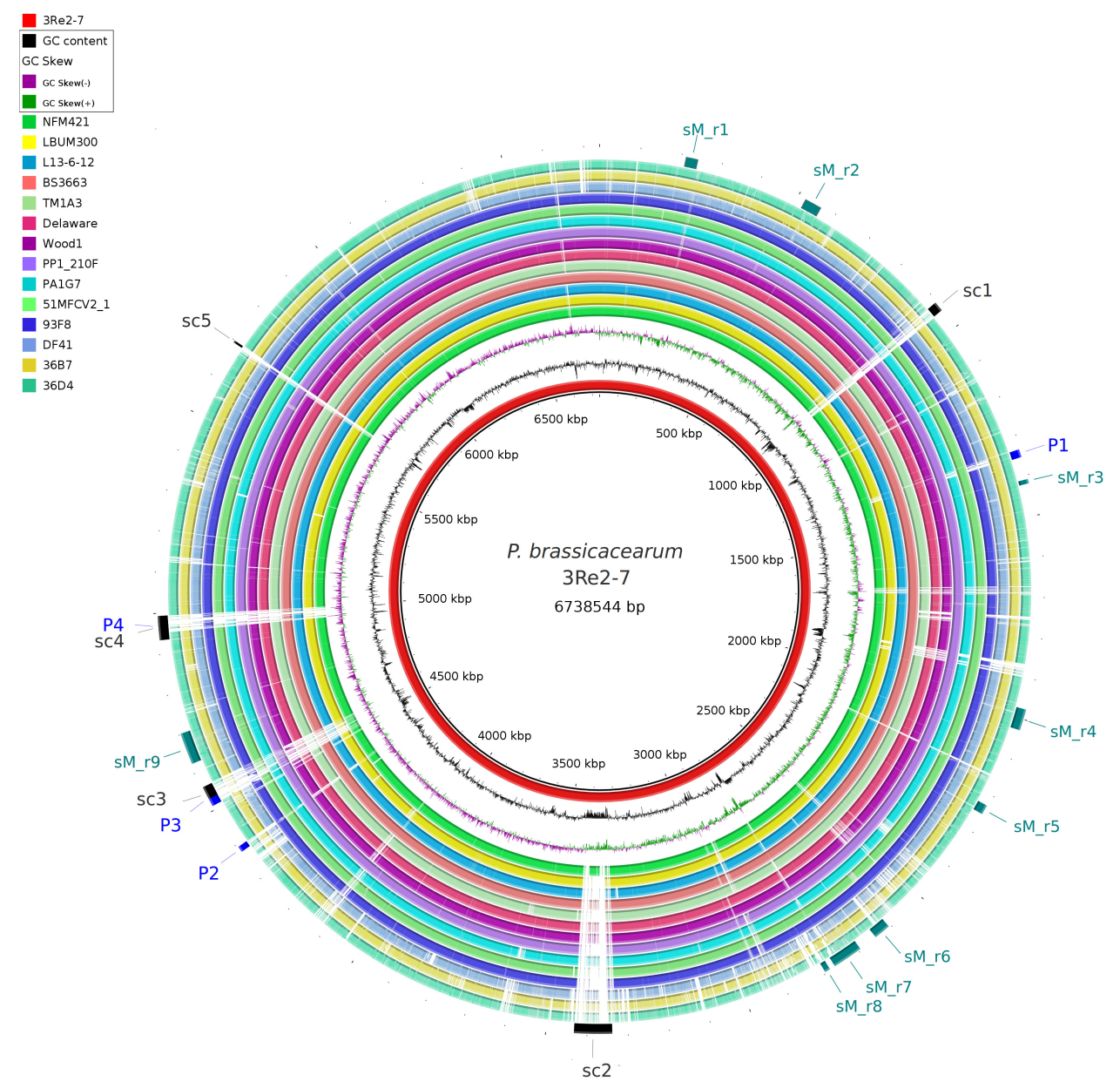

Figure 2. BLAST comparison of P. brassicacearum genomes. Circular representation of the similarity between P. brassicacearum genomes listed in Table 1 with strain 3Re2-7 as reference (black innermost ring). The innermost graphs depict GC content (black) and GC skew (purple/green) followed by concentric rings of query sequences colored according to BLAST identity; gaps in the rings represent regions of low or no similarity. On the outermost ring, clusters of 3Re2-7 specific genes are indicated in black, abbreviated with sc* (Singleton cluster 1-5), gene regions related to biosynthesis of secondary metabolites detected with antiSMASH are indicated in teal and abbreviated with sM_r* (secondaryMetabolism_region 1-9) and detected phage sequences in blue (P1-P4). BLAST analysis and generation of this comparative view was done using BRIG [31]. 


\subsection{Taxonomic Classification Based on Whole-Genome and Core Gene Analyses}

In 2002, the strain 3Re2-7 was first classified as Pseudomonas reactans by fatty acid methyl ester analysis and 16S rDNA sequencing, and then in 2004 reclassified according to its $16 S$ rDNA sequence as $P$. trivialis $[25,26,32]$. The intrageneric similarity of $16 \mathrm{~S}$ rRNA gene sequences between species of the genus Pseudomonas is very high (>98.5\%, [33]). Therefore, the resolution of 16S rRNA gene sequence similarity values is not high enough for Pseudomonas species delineation [11,34]. Based on the whole genome sequence, we revised the taxonomic classification of the strain 3Re2-7 to P. brassicacearum using the high-resolution classification approaches of Average Amino-acid Identity (AAI) and in silico DNA-DNA hybridization (DDH). These analyses showed, that AAI values are at $85.00 \%$ and $99.59 \%$ for identities with P. trivialis DSM $14937^{T}$ and P. brassicacearum subsp. brassicacearum NFM421, respectively. Accordingly, the in silico DDH estimate is at $25.30 \%$ with respect to $P$. trivialis DSM $14937^{T}$ and $96.10 \%$ to P. brassicacearum subsp. brassicacearum NFM421. Therefore, the strain 3 Re2-7 can be classified as P. brassicacearum rather than $P$. trivialis.

Further, pairwise Average Amino-acid Identities (AAIs) of all publicly available P. brassicacearum strains (as listed in Table 1) were calculated. Additionally, all publicly available P. kilonensis strains were included in the AAI analysis, since P. kilonensis has previously been suggested as the 'junior synonym' to P. brassicacearum [35]. As shown in Figure 3, calculated pairwise AAIs show values of $99.8 \%$ and higher for twelve very closely related strains including the strain 3Re2-7 and the representative P. brassicacearum strain NFM421 (cluster I including TM1A3, Delaware, Wood1, BS3663, 3Re2-7, PP1_210F, L13-6-12, PA1G7, LBUM300, 51MFCVI2.1, 93F8 and NFM421).

The P. kilonensis strains form a distinct cluster (Figure 3, cluster II) but feature high AAI values of $97.5 \%$ and higher to cluster I.

A third separate cluster is formed by the strains DF41, 36B7 and 36D4 (Figure 3, cluster III). These three strains are more distinct from the highly similar P. brassicacearum and P. kilonensis clusters but still above the proposed 95\% species threshold [36,37]. Even though AAI values of strains LZ_4 and Wood3 to the representative P. brassicacearum strain NFM421 are slightly below the species threshold, they are still relatively high $(94.9 \%$ and $94.7 \%$, respectively, Figure 3, cluster IV). They might still be considered as P. brassicacearum species. In contrast, AAI values of the strains 37D10, 48H11, 38D7 and 38D4 to the P. brassicacearum representative strain are clearly below the 95\% species threshold (Figure 3, group V) and therefore their species allocation should be rethought. Thus, these four strains were not included in the detailed comparative analysis.

The constructed phylogenetic tree (Figure 4) based on 2331 core genes shows the position of the analyzed P. brassicacearum strains relative to type strains of other biocontrol Pseudomonas species (P. fluorescens DSM 50090 ${ }^{T}$, P. veronii DSM $11331^{T}$, P. azotoformans NBRC $12693^{T}$, P. trivialis DSM $14937^{T}$, P. orientalis DSM $17489^{T}$ and P. synxantha NBRC $3913^{T}$ ). A distinct cluster is formed by the P. brassicacearum species, which as expected includes the P. kilonensis strains. The subclusters that emerged in the AAI analysis also appear in the phylogenetic tree (Figure 4).

Interestingly, P. brassicacearum strains with very similar GC contents clustered together independently of the method used (Table 1, Figures 3 and 4). This observation suggests an evolutionary association of GC content and phylogenetic distance within the species. The exceptional high similarity between P. brassicacearum strains may reflect adaptations to similar environmental conditions. Unfortunately, metadata reporting is inconsistent. For some strains the exact origin is reported unspecifically or unknown. Consistent and specific metadata would allow us to draw conclusions or formulate hypotheses regarding niche adaptation. 
Table 1. General genome features of publicly available genomes of Pseudomonas brassicacearum and P. kilonensis strains. https://www.ncbi.nlm.nih.gov/genome/ genomes/3640, last accessed 13.06.2019.

\begin{tabular}{|c|c|c|c|c|c|c|c|c|}
\hline Organism/Name & Strain & Size $[\mathrm{Mb}]$ & GC [\%] & Scaf-Folds & Genes & Niche/Source & References & $\mathrm{AAI}^{a}$ \\
\hline P. brassicacearum & $3 \operatorname{Re} 2-7$ & 6.7 & 60.8 & 1 & 6267 & Potato endorhiza & [32], this study & 99.9 \\
\hline P. brassicacearum subsp. brassicacearum & NFM421 ${ }^{b}$ & 6.8 & 60.8 & 1 & 6209 & Arabidopsis thaliana rhizoplane & {$[16,18]$} & 100 \\
\hline P. brassicacearum & DF41 & 6.6 & 60.5 & 1 & 5884 & Canola root & [19] & 96 \\
\hline P. brassicacearum & LBUM300 & 6.9 & 60.8 & 1 & 6308 & Strawberry rhizosphere & [22] & 99.9 \\
\hline P. brassicacearum & L13-6-12 & 6.7 & 60.9 & 1 & 6046 & Potato rhizosphere & {$[21]$} & 99.9 \\
\hline P. brassicacearum & BS3663 & 6.7 & 60.8 & 1 & 6129 & No metadata available & Unpublished & 99.9 \\
\hline P. brassicacearum & 51MFCVI2.1 & 6.5 & 61.0 & 49 & 5980 & Rhizosphere & Unpublished & 99.9 \\
\hline P. brassicacearum & PP1_210F & 6.7 & 60.8 & 5 & 6159 & Peels tuber & {$[20,38]$} & 99.9 \\
\hline P. brassicacearum & PA1G7 & 6.7 & 60.8 & 8 & 6180 & Potato field soil & {$[20,38]$} & 99.9 \\
\hline P. brassicacearum & LZ-4 & 6.2 & 60.1 & 111 & 5642 & Yellow River & Unpublished & 94.9 \\
\hline P. brassicacearum & TM1A3 & 6.6 & 60.9 & 29 & 6037 & Tomato rhizosphere & [39] & 99.8 \\
\hline P. brassicacearum & Wood3 & 6.3 & 62.3 & 433 & 5752 & Agricultural & Unpublished & 94.7 \\
\hline P. brassicacearum & $36 \mathrm{~B} 7$ & 7.1 & 60.7 & 28 & 6411 & Agricultural & Unpublished & 95.8 \\
\hline P. brassicacearum & 36D4 & 7.0 & 60.5 & 128 & 6290 & Agricultural & Unpublished & 95.8 \\
\hline P. brassicacearum & Delaware & 6.8 & 60.8 & 226 & 6263 & Agricultural & Unpublished & 99.8 \\
\hline P. brassicacearum & $93 \mathrm{~F} 8$ & 6.8 & 61.0 & 36 & 6291 & Agricultural & Unpublished & 99.9 \\
\hline P. brassicacearum & 37D10 & 6.3 & 58.8 & 55 & 5946 & Agricultural & Unpublished & 88.6 \\
\hline P. brassicacearum & Wood1 & 6.9 & 60.8 & 187 & 6288 & Agricultural & Unpublished & 99.8 \\
\hline P. brassicacearum & $48 \mathrm{H} 11$ & 6.0 & 58.8 & 36 & 5510 & Agricultural & Unpublished & 88.7 \\
\hline P. brassicacearum & $38 \mathrm{D} 7$ & 6.5 & 59.5 & 485 & 6087 & Agricultural & Unpublished & 89.2 \\
\hline P. brassicacearum & 38D4 & 7.1 & 58.7 & 199 & 6793 & Agricultural & Unpublished & 89.5 \\
\hline P. kilonensis & $1855-344$ & 6.8 & 60.7 & 73 & 6146 & Soil & [40] & 98.2 \\
\hline P. kilonensis & P12 & 6.4 & 60.8 & 44 & 5791 & Tobacco & [39] & 98.2 \\
\hline P. kilonensis & $\mathrm{DSM} 13647^{T}$ & 6.4 & 60.9 & 44 & 5758 & No metadata available & Unpublished & 98.1 \\
\hline P. kilonensis & ACN7 & 6.5 & 61 & 200 & 5897 & Compost soil & [41] & 98.2 \\
\hline P. kilonensis & ACN4 & 6.5 & 60.8 & 91 & 5956 & Compost soil & [41] & 98.2 \\
\hline P. kilonensis & ZKA7 & 6.8 & 60.6 & 1 & 6158 & No metadata available & Unpublished & 97.9 \\
\hline P. kilonensis & BS3780 & 6.4 & 60.8 & 2 & 5775 & No metadata available & Unpublished & 98.1 \\
\hline
\end{tabular}

${ }^{a}$ Average Amino-acid Identity (AAI) with the representative strain of the P. brassicacearum species, NFM421; ${ }^{b}$ Representative strain. 


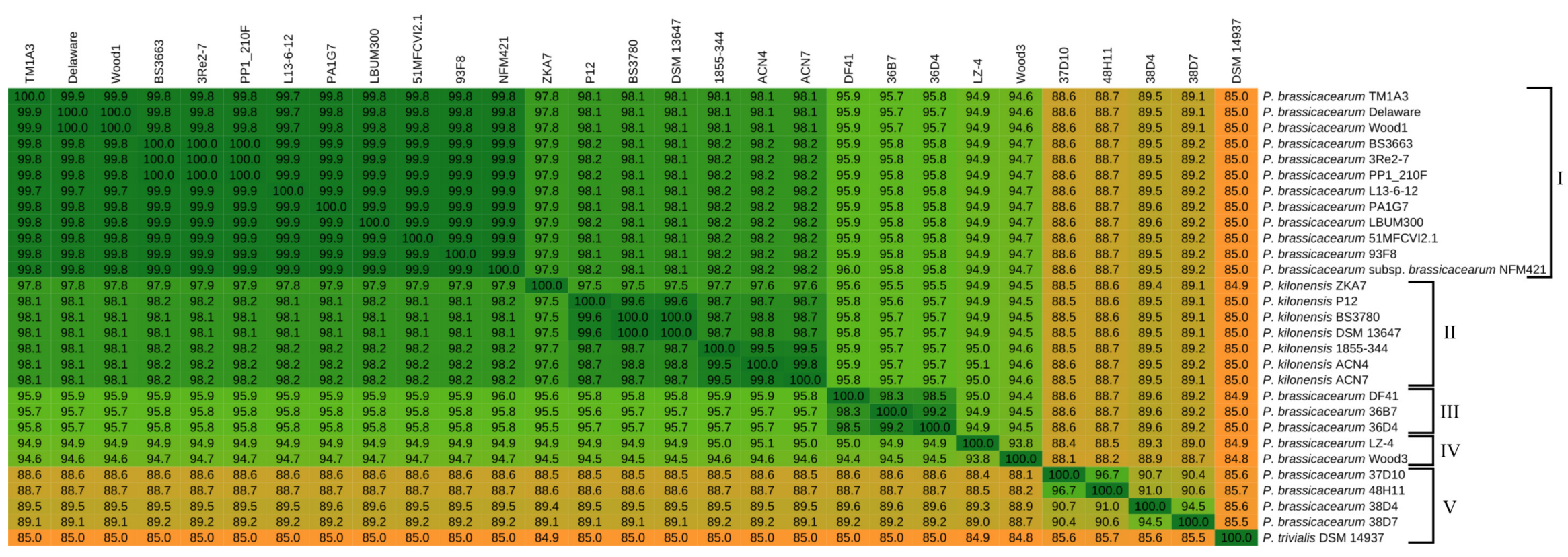

Figure 3. Average Amino-acid Identity (AAI) matrix of genome sequenced P. brassicacearum and P. kilonensis strains. Emerging clusters/groups are indicated with brackets and numbered I-V. The protein sequences of orthologous core genes of the genomes were analyzed for their mean percent identity values. The values were calculated within the EDGAR2.0 platform [42]. 
0.01

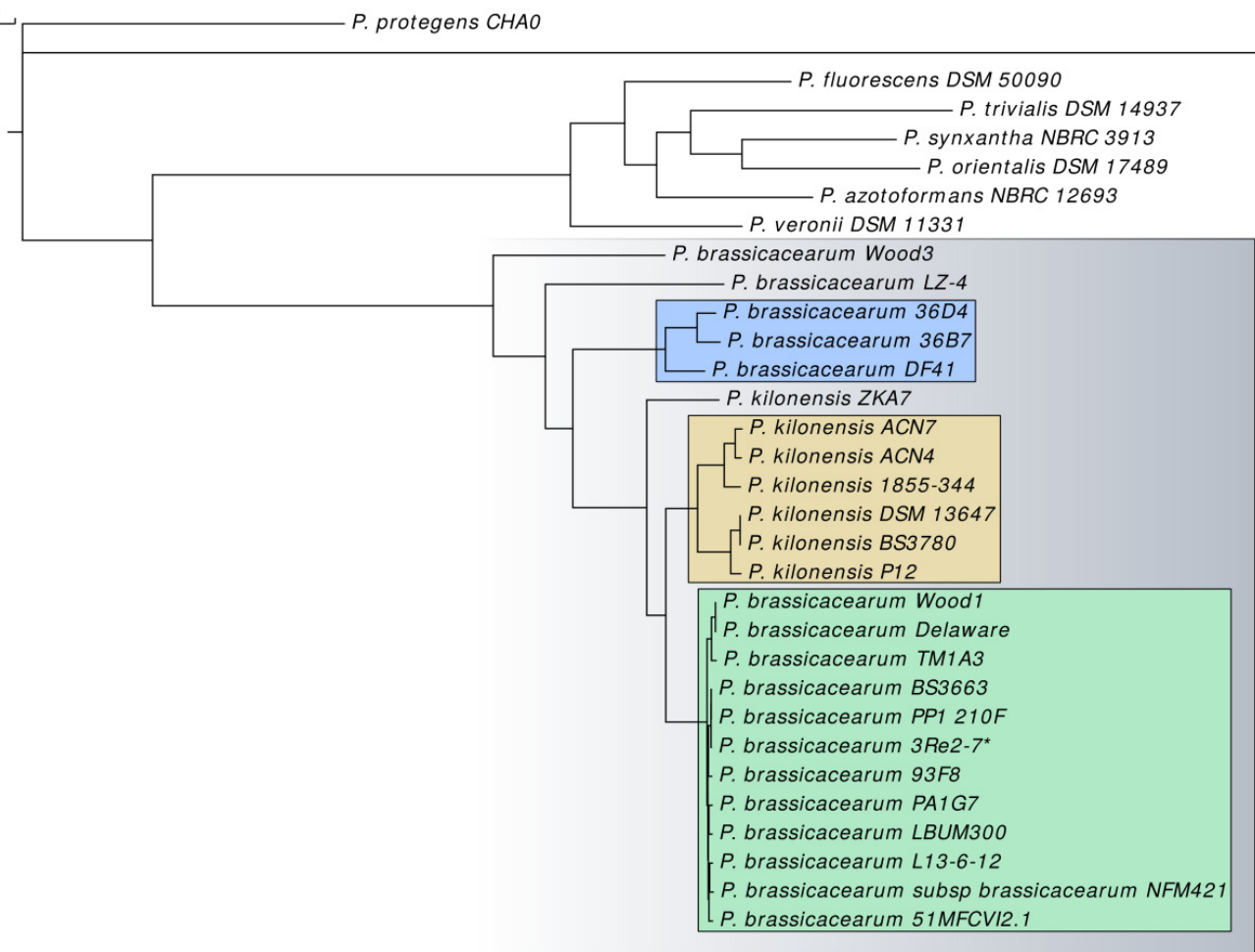

Figure 4. The phylogenetic relationship of P. brassicacearum species relative to other biocontrol Pseudomonas species. As outgroup, the type strain of the genus, P. aeruginosa DSM 50071 ${ }^{T}$, was included. The tree is based on 2331 core gene products. Selected clusters are indicated by colored boxes. Strain P. brassicacearum 3Re2-7 studied here is marked with an asterisk. The phylogenetic analysis was performed within the EDGAR 2.0 platform [42]. The bar indicates 1 substitutions per 100 positions. 


\subsection{Comparative Genomics Revealed Genes Unique to P. brassicacearum 3Re2-7}

To assess the unique genetic potential of strain 3Re2-7, a comparative analysis including publicly available genomes of in total 16 P. brassicacearum strains confirmed by AAI analyses was performed using the software platform EDGAR [42]. The core genome of the P. brassicacearum species consists of 3556 CDSs with P. brassicacearum subsp. brassicacearum NFM421 used as reference. For P. brassicacearum 3Re2-7, 105 unique genes without any orthologous genes in the compared genomes were detected. Of these 105 singletons, 78 were annotated as genes encoding hypothetical proteins and 27 have predicted functions. Some of these singletons are clustered within the genome (see their regions indicated in Figure 2). During the detailed analysis, five regions emerged, in which clustered singletons occur. A striking deviation in the GC content to the remaining genome was observed in the genomic regions where these clusters are localized (Figure 2). These regions may represent functional clusters of so far unknown function. The smallest cluster comprises four singleton genes and the largest 25 . Cluster_3 and cluster_4 correspond to the location of the detected prophage regions P3 and P4, respectively.

Table 2. Annotation of P. brassicacearum 3Re2-7 singleton cluster_1.

\begin{tabular}{ll}
\hline Locus tag & Predicted Function \\
\hline ELZ14_04045 & hypothetical protein \\
ELZ14_04050 & DNA-binding protein \\
ELZ14_04055 & hypothetical protein \\
ELZ14_04060 & hypothetical protein \\
ELZ14_04065 & hypothetical protein \\
ELZ14_04070 & hypothetical protein \\
ELZ14_04075 & nucleotidyltransferase family protein \\
ELZ14_04080 * & hypothetical protein \\
ELZ14_04085 * & MafI family immunity protein \\
ELZ14_04090 & hypothetical protein \\
ELZ14_04095 & hypothetical protein \\
ELZ14_04100 & hypothetical protein \\
ELZ14_04105 & DUF4935 domain-containing protein \\
ELZ14_04110 & hypothetical protein \\
ELZ14_04115* & hypothetical protein \\
ELZ14_04120 & hypothetical protein \\
ELZ14_04125 * & hypothetical protein \\
ELZ14_04130 & hypothetical protein \\
ELZ14_04135 * & integrase \\
ELZ14_04140 & hypothetical protein \\
ELZ14_04145 & hypothetical protein \\
ELZ14_04150 & hypothetical protein \\
ELZ14_04155 & hypothetical protein \\
ELZ14_04160 & metallohydrolase \\
\hline & * Non-singleton genes.
\end{tabular}

Cluster_1 comprises 13 singleton genes that encode hypothetical proteins with unknown function and four singleton genes with the functions listed in Table 2. Metallohydrolases are enzymes that catalyze the hydrolysis of ester and amide bonds [43], while nucleotidyltransferases transfer nucleotides from one compound to another [44]. An adenylating protein similar to ThiF has been shown to be involved in the biosynthesis of the unusual siderophore thioquinolobactin in Pseuodomonas fluorescens ATCC17400 [45]. The DUF4935 domain shows homology to other PIN-like families and is found in the N-terminal region of uncharacterized proteins. It contains several conserved acidic residues critical for chelating metal ions [46]. In the genetic context, also a non-singleton ferric iron uptake transcriptional regulator gene (ELZ14_04245) is present. The regulator was shown to repress and also activate siderophore synthesis in pathogens [47]. According to IslandViewer 4 [30], the region enclosing cluster_1 is a genomic island (supported by three of four prediction methods). In between the singleton genes, a non-singleton gene encoding an integrase (ELZ14_04135) is located. Integrases are known to 
mediate integration of integrative conjugative elements (ICEs; a.k.a. conjugative transposons) [48]. ICEs are modular mobile genetic elements (MGEs) integrated into a host genome and have a broad range of associated phenotypes, for example, antibiotic resistance, symbiosis, pathogenesis, bacteriocin synthesis and biofilm formation [48]. Further, there is evidence for a toxin-antitoxin system encoded in cluster_1 (ELZ14_04230, ELZ14_03880-03895). A toxin-antitoxin system is an accessory function typical of MGEs that most probably promotes the maintenance of an ICE [49]. Due to these findings, it could be possible that this putative MGE encodes enzymes for the synthesis of an unknown siderophore.

Table 3. Annotation of P. brassicacearum 3Re2-7 singleton cluster_2.

\begin{tabular}{|c|c|}
\hline Locus Tag & Predicted Function \\
\hline ELZ14_15030 & hypothetical protein \\
\hline ELZ14_15035 * & hypothetical protein \\
\hline ELZ14_15040 & helicase IV \\
\hline ELZ14_15045 * & HNH endonuclease \\
\hline ELZ14_15050 & hypothetical protein \\
\hline ELZ14_15055 & endonuclease \\
\hline ELZ14_15060 & hypothetical protein \\
\hline ELZ14_15065* & hypothetical protein \\
\hline ELZ14_15070 & hypothetical protein \\
\hline ELZ14_15075 & hypothetical protein \\
\hline ELZ14_15080 & hypothetical protein \\
\hline ELZ14_15085 & CHAT domain-containing protein \\
\hline ELZ14_15090 & hypothetical protein \\
\hline ELZ14_15095 * & DNA/RNA non-specific endonuclease \\
\hline ELZ14_15100 * & hypothetical protein \\
\hline ELZ14_15105* & relaxase \\
\hline ELZ14_15110 & DUF3742 family protein \\
\hline ELZ14_15115 * & conjugal transfer protein TraG \\
\hline ELZ14_15120 * & integrating conjugative element protein \\
\hline ELZ14_15125 * & TIGR03756 family integrating conjugative element protein \\
\hline ELZ14_15130* & DNA repair protein RadC \\
\hline ELZ14_15135 & hypothetical protein \\
\hline (...) $32^{*}$ & \\
\hline ELZ14_15295 & HAD family hydrolase \\
\hline ELZ14_15300 & DNA-processing protein DprA \\
\hline ELZ14_15305 * & hypothetical protein \\
\hline ELZ14_15310 & hypothetical protein \\
\hline ELZ14_15315 & DUF4935 domain-containing protein \\
\hline ELZ14_15320 & hypothetical protein \\
\hline ELZ14_15325 & DUF3800 domain-containing protein \\
\hline ELZ14_15330 & toll/interleukin-1 receptor domain-containing protein \\
\hline ELZ14_15335 & RNA-directed DNA polymerase \\
\hline ELZ14_15340 & hypothetical protein \\
\hline ELZ14_15345 *,a & hypothetical protein \\
\hline ELZ14_15350 *,a & transposase \\
\hline ELZ14_15355 & hypothetical protein \\
\hline ELZ14_15360 * & hypothetical protein \\
\hline ELZ14_15365 & hypothetical protein \\
\hline ELZ14_15370 * & hypothetical protein \\
\hline ELZ14_15375 & ATP-binding protein \\
\hline ELZ14_15380 * & hypothetical protein \\
\hline ELZ14_15385 *,a & leucine $\mathrm{ABC}$ transporter subunit substrate-binding protein LivK \\
\hline ELZ14_15390 & hypothetical protein \\
\hline
\end{tabular}

The largest cluster (cluster_2) includes 14 hypothetical proteins and 11 annotated genes with the functions listed in Table 3. Helicase IV catalyses ATP dependent unwinding of double stranded DNA to single stranded DNA [50], while endonucleases are enzymes that cleave the phosphodiester 
bond within a polynucleotide chain. A relation of some proteins containing the CHAT (Caspase HetF Associated with Tprs) domain to peptidases in peptidase clan CD that includes the caspases is known [51,52]. The DUF3742 domain family is found in bacteria and is of so far unknown function [51]. The haloacid dehalogenase-like hydrolase (HAD) superfamily is a large group of proteins with diverse substrate specificities whose members are involved in the cleavage of carbon-halogen bonds, phosphatase, phosphonatase and phosphoglucomutase reactions [53]. RNA-directed DNA polymerase (reverse transcriptase) occurs in a variety of mobile elements, including prokaryotic retroelements and bacterial msDNAs [51]. The enzyme synthesizes DNA on a RNA template for integration into the host genome and exploitation of a host cell [51]. Interestingly, this cluster was reported as genomic island supported by four prediction methods integrated in IslandViewer 4 [30]. Some genes included in this region (not reported as singletons, ELZ14_15120, ELZ14_15125, ELZ14_15150-75, ELZ14_15190-205 and ELZ14_15250) have a function associated with integrative conjugative elements (ICEs). Also a relaxase (ELZ14_15105) is encoded in cluster_2. Relaxases perform the initial step in the ICE transfer mechanism [48]. Type I and type VI secretion as well as type IV conjugative transfer system components are also present in the region of singleton cluster_2 (ELZ14_14955, ELZ14_15020, ELZ14_15025 and ELZ14_15195) and suggest the functionality of the putative ICE [48]. Taken together these results with the predicted protein functions, cluster_ 2 might be of horizontal origin and have a possible function in the biocontrol context and contribute to bacterial fitness and competitiveness in the endorhiza.

The small cluster_5 shown in Table 4 was predicted to encode two hypothetical proteins (ELZ14_26355 and 26360), a DNA helicase (ELZ14_26365) and a GNAT family N-acetyltransferase (ELZ14_26370). DNA-helicase unwinds the two strands in a DNA double helix. GCN5-related $\mathrm{N}$-acetyltransferases family (GNAT) N-acetyltransferases transfer an acetyl group from acetyl-CoA to a large array of substrates, from small molecules such as aminoglycoside antibiotics to macromolecules [54]. These singletons also are located in a region predicted as genomic island by IslandViewer 4 [30] (supported by three prediction methods). A functional prediction for this region can not be deduced.

Table 4. Annotation of P. brassicacearum 3Re2-7 singleton cluster_5.

\begin{tabular}{ll}
\hline Locus Tag & Predicted Function \\
\hline ELZ14_26355 & hypothetical protein \\
ELZ14_26360 & hypothetical protein \\
ELZ14_26365 & DNA helicase \\
ELZ14_26370 & GNAT family N-acetyltransferase \\
\hline
\end{tabular}

P. brassicacearum 3Re2-7 was isolated from the endorhiza of potato and therefore most probably has evolved mechanisms to sustain in competitive environments like soil, rhizosphere and endorhiza. By combining genome mining with comparative genomics, we were able to find gene clusters specific to strain 3Re2-7 with the potential to be related to niche adaptation or biocontrol. Our results show, that even though the genomes of $P$. brassicacearum strains are very similar, they still have unique traits that are interesting targets for the detection of new microbial gene clusters of biotechnological potential, that need experimental confirmation.

\subsection{Genome Mining of P. brassicacearum 3Re2-7 Revealed Biocontrol Determinants}

To examine putative genetic determinants involved in biocontrol characteristics of $P$. brassicacearum 3Re2-7, different genome-mining tools were applied. Putative biocontrol genes involved in the biocontrol activity of this strain were identified in the genome and are discussed in the following sections. Genes predicted to encode functions in secondary metabolite and antibiotic biosynthesis (Table 5), induced systemic resistance and plant growth promotion (Table 6), pathogen inhibition (Table 7) and other traits of importance in a competitive environment (Table 8) were identified. The localization of selected genetic features in the 3Re2-7 genome is indicated in Figure 2. 
Table 5. Genetic biocontrol determinants of P. brassicacearum 3Re2-7-Secondary Metabolism and Antibiotics.

\begin{tabular}{|c|c|c|c|}
\hline Biocontrol Trait & Locus Tag (Gene Name) & Predicted EC Number and Protein Function & $\log 2\left(\mathrm{TPM}^{a}\right)$ \\
\hline \multicolumn{4}{|c|}{ Secondary metabolism \& antibiotics } \\
\hline \multirow{8}{*}{ DAPG $^{b}$ synthesis } & ELZ14_11930 (phlE) & n.a.; MFS transporter & 8.18 \\
\hline & ELZ14_11935 (phlD) & EC 2.3.1.-; type III polyketide synthase & 11.05 \\
\hline & ELZ14_11940 (phlB) & n.a.; 2,4-diacetylphloroglucinol biosynthesis protein & 10.12 \\
\hline & ELZ14_11945 (phlC) & n.a.; thiolase family protein & 10.79 \\
\hline & ELZ14_11950 (phlA) & EC 2.3.3.10; hydroxymethylglutaryl-CoA synthase & 10.86 \\
\hline & ELZ14_11955 (phlF) & n.a.; TetR/AcrR family transcriptional regulator & 5.23 \\
\hline & ELZ14_11960 (phlG) & n.a.; 2,4-diacetylphloroglucinol hydrolase & 10.97 \\
\hline & ELZ14_11965 (phlH) & n.a.; TetR/AcrR family transcriptional regulator & 7.07 \\
\hline \multirow{3}{*}{$\mathrm{HCN}^{c}$ synthesis } & ELZ14_17910 (hcnC) & EC 1.4.99.5; cyanide-forming glycine dehydrogenase subunit HcnC & 9.33 \\
\hline & ELZ14_17915 (hcnB) & EC 1.4.99.5; cyanide-forming glycine dehydrogenase subunit HcnB & 8.95 \\
\hline & ELZ14_17920 (hcnA) & EC 1.4.99.5; cyanide-forming glycine dehydrogenase subunit HcnA & 9.89 \\
\hline
\end{tabular}

${ }^{a}$ Transcript Per Million; ${ }^{b}$ 2,4-diacetylphloroglucinol; ${ }^{c}$ Hydrogen cyanide. 
Table 6. Genetic biocontrol determinants of P. brassicacearum 3Re2-7-Induced systemic resistance \& Plant growth promotion.

\begin{tabular}{|c|c|c|c|}
\hline Biocontrol Trait & Locus Tag (Gene Name) & Predicted EC Number and Protein Function & $\log 2\left(\mathrm{TPM}^{a}\right)$ \\
\hline \multirow{8}{*}{ Volatiles } & ELZ14_17085 (-) & EC 1.1.1.4; 2,3-butanediol dehydrogenase & 4.47 \\
\hline & ELZ14_17090 (acoC) & EC 2.3.1.12; acetoin dehydrogenase dihydrolipoyllysine-residue acetyltransferase subunit & 4.51 \\
\hline & ELZ14_17095 (асов) & EC 1.1.1.-; alpha-ketoacid dehydrogenase subunit beta & 4.55 \\
\hline & ELZ14_17100 (acoA) & EC 1.1.1.-; thiamine pyrophosphate-dependent dehydrogenase E1 component subunit alpha & 4.58 \\
\hline & ELZ14_26505 (ilvC) & EC 1.1.1.86; Ketol-acid reductoisomerase & 8.25 \\
\hline & ELZ14_26510 (ilvH) & EC 2.2.1.6; Acetolactate synthase isozyme 3 small subunit & 6.78 \\
\hline & ELZ14_26515 (ilvI) & EC 2.2.1.6; Acetolactate synthase isozyme 3 large subunit & 6.13 \\
\hline & ELZ14_12055 (ilvX) & EC 2.2.1.6; Putative acetolactate synthase large subunit IlvX & 1.81 \\
\hline \multirow{14}{*}{ Plant growth promotion } & ELZ14_11285 (acdS) & EC 3.5.99.7; 1-aminocyclopropane-1-carboxylate deaminase & 4.66 \\
\hline & ELZ14_14325 (ribBA) & n.a.; 3,4-dihydroxy-2-butanone-4-phosphate synthase/GTP cyclohydrolase II & 1.98 \\
\hline & ELZ14_23780 (ribH) & EC 2.5.1.78; 6,7-dimethyl-8-ribityllumazine synthase & 4.92 \\
\hline & ELZ14_26870 (ribF) & EC 2.7.1.26; bifunctional riboflavin kinase/FAD synthetase & 5.40 \\
\hline & ELZ14_27845 (ribA) & EC 3.5.4.25; GTP cyclohydrolase II & 5.63 \\
\hline & ELZ14_27870 (ribH2) & EC 2.5.1.78; 6,7-dimethyl-8-ribityllumazine synthase & 8.05 \\
\hline & ELZ14_27875 (ribB) & n.a.; 3,4-dihydroxy-2-butanone-4-phosphate synthase & 4.82 \\
\hline & ELZ14_27880 (ribE) & EC 2.5.1.9; riboflavin synthase & 4.69 \\
\hline & ELZ14_27885 (ribD) & n.a.; riboflavin biosynthesis protein RibD & 3.80 \\
\hline & ELZ14_28390 (trpC) & EC 4.1.1.48; indole-3-glycerol phosphate synthase TrpC & 6.23 \\
\hline & ELZ14_28395 (trpD) & EC 2.4.2.18; anthranilate phosphoribosyltransferase & 5.98 \\
\hline & ELZ14_28400 (trpG) & EC 4.1.3.27; aminodeoxychorismate/anthranilate synthase component II & 5.02 \\
\hline & ELZ14_28405 (est $A)$ & EC 3.1.1.1; autotransporter domain-containing esterase & 3.90 \\
\hline & ELZ14_28410 (trpE) & EC 4.1.3.27; anthranilate synthase component I & 4.43 \\
\hline \multirow{15}{*}{ Phosphate solubilization } & ELZ14_15680 (phyC) & EC 3.1.3.8; phytase & 0.18 \\
\hline & ELZ14_27855 (pgpA) & EC 3.1.3.27; phosphatidylglycerophosphatase A & 6.49 \\
\hline & ELZ14_28415 $(g p h)$ & EC 3.1.3.18; phosphoglycolate phosphatase & 4.60 \\
\hline & ELZ14_28615 (pqqF) & EC:3.4.24.-; pyrroloquinoline quinone biosynthesis protein $\mathrm{PqqF}$ & 3.98 \\
\hline & ELZ14_28620 (pqqA) & n.a.; pyrroloquinoline quinone precursor peptide PqqA & 12.77 \\
\hline & ELZ14_28625 (pqqB) & n.a.; pyrroloquinoline quinone biosynthesis protein PqqB & 6.86 \\
\hline & ELZ14_28630 (pqqC) & EC 1.3.3.11; pyrroloquinoline-quinone synthase PqqC & 5.99 \\
\hline & ELZ14_28635 (pqqD) & n.a.; pyrroloquinoline quinone biosynthesis peptide chaperone PqqD & 6.32 \\
\hline & ELZ14_28640 (pqqE) & n.a.; pyrroloquinoline quinone biosynthesis protein $\mathrm{PqqE}$ & 5.96 \\
\hline & ELZ14_30735 (-) & EC 3.1.3.16; serine/threonine-protein phosphatase & 5.10 \\
\hline & ELZ14_08470 (mирP) & EC 3.1.3.105; N-acetylmuramic acid 6-phosphate phosphatase MupP & 7.05 \\
\hline & ELZ14_08475 (ubiG) & EC 2.1.1.222; 3-demethylubiquinol 3-O-methyltransferase UbiG & 7.36 \\
\hline & ELZ14_04440 (-) & EC 3.1.3.1; alkaline phosphatase & 6.63 \\
\hline & ELZ14_04880 (-) & EC 3.1.3.1; alkaline phosphatase family protein & 2.57 \\
\hline & ELZ14_09815 (-) & EC 3.1.3.16; phosphoprotein phosphatase & 3.15 \\
\hline
\end{tabular}

${ }^{a}$ Transcript Per Million. 
Table 7. Genetic biocontrol determinants of P. brassicacearum 3Re2-7 - Pathogen inhibition.

\begin{tabular}{llll}
\hline Biocontrol Trait & Locus Tag (Gene Name) & Predicted EC Number and Protein Function & log2 (TPM ${ }^{a}$ ) \\
\hline & ELZ14_01095 (-) & n.a.; TonB-dependent siderophore receptor & 0.32 \\
& ELZ14_04245 (fur) & n.a.; ferric iron uptake transcriptional regulator & 8.94 \\
& ELZ14_10605 (-) & n.a.; TonB-dependent siderophore receptor & 1.14 \\
& ELZ14_12290 (hcsK) & n.a.; siderophore-iron reductase FhuF & 4.09 \\
& ELZ14_13330 (pchB) & n.a.; isochorismate lyase & 4.10 \\
& ELZ14_14280 (-) & n.a.; TonB-dependent siderophore receptor & 2.35 \\
Iron acquisition & ELZ14_16995 (-) & n.a.; TonB-dependent siderophore receptor & 1.85 \\
& ELZ14_18660 (-) & n.a.; TonB-dependent siderophore receptor & 0.84 \\
& ELZ14_21210 (pvdA) & EC 1.14.13.196; ornithine monooxygenase & 1.30 \\
& ELZ14_21260 (-) & n.a.; nonribosomal peptide synthetase & 1.42 \\
& ELZ14_21280 (-) & n.a.; TonB-dependent siderophore receptor & 2.82 \\
& ELZ14_21285 (-) & n.a.; nonribosomal peptide synthetase & 3.41 \\
& ELZ14_24950 (-) & n.a.; siderophore-interacting protein & 4.42 \\
& ELZ14_26060 (-) & n.a.; TonB-dependent siderophore receptor & 0.26 \\
& ELZ14_28775 (-) & n.a.; TonB-dependent siderophore receptor & 0.46 \\
\hline & ELZ14_14600 (-) & n.a.; matrixin family metalloprotease & 12.59 \\
& ELZ14_14610 (prsD) & n.a.; type I secretion system permease/ATPase PrsD & 5.81 \\
& ELZ14_14615 (-) & n.a.; HlyD family type I secretion periplasmic adaptor subunit & 6.32 \\
& ELZ14_14620 (-) & n.a.; peptidase & 4.73 \\
& ELZ14_20130 (-) & n.a.; Hcp family type VI secretion system effector & 1.77 \\
\hline Choprotease activitinase activity & ELZ14_25035 (nagA) & EC 3.5.1.25; N-acetylglucosamine-6-phosphate deacetylase & 2.72 \\
\hline
\end{tabular}

${ }^{a}$ Transcript Per Million. 
Table 8. Genetic biocontrol determinants of P. brassicacearum 3Re2-7-Others.

\begin{tabular}{|c|c|c|c|}
\hline Biocontrol Trait & Locus Tag (Gene Name) & Predicted EC Number and Protein Function & $\log 2\left(\mathrm{TPM}^{a}\right)$ \\
\hline \multirow{7}{*}{ Metabolism } & ELZ14_02400 (gltB) & EC 1.4.1.13; glutamate synthase large subunit & 5.12 \\
\hline & ELZ14_02405 (gltD1) & EC 1.4.1.13; glutamate synthase small subunit & 5.59 \\
\hline & ELZ14_02380 (aroK) & EC 2.7.1.71; shikimate kinase AroK & 5.44 \\
\hline & ELZ14_02385 (aroB) & EC 4.2.3.4; 3-dehydroquinate synthase & 5.04 \\
\hline & ELZ14_03505 (aroQ) & EC 4.2.1.10; type II 3-dehydroquinate dehydratase & 5.88 \\
\hline & ELZ14_08510 (aroA) & EC 2.5.1.19; 3-phosphoshikimate 1-carboxyvinyltransferase & 6.40 \\
\hline & ELZ14_11900 (-) & n.a.; polyketide cyclase & 2.67 \\
\hline \multirow{8}{*}{ Exopolysaccharides } & ELZ14_25290 (algD) & n.a.; nucleotide sugar dehydrogenase & 9.65 \\
\hline & ELZ14_25300 (alg44) & n.a.; alginate biosynthesis protein $\mathrm{Alg} 44$ & 7.31 \\
\hline & ELZ14_25320 (algX) & n.a.; alginate $\mathrm{O}$-acetyltransferase & 7.49 \\
\hline & ELZ14_25325 (algL) & EC 4.2.2.3; mannuronate-specific alginate lyase & 7.89 \\
\hline & ELZ14_25335 (algJ) & n.a.; alginate $\mathrm{O}$-acetyltransferase & 7.22 \\
\hline & ELZ14_25340 (algF) & n.a.; alginate $\mathrm{O}$-acetyltransferase & 8.93 \\
\hline & ELZ14_25350 (algA) & n.a.; alginate biosynthesis protein $\mathrm{Alg} A$ & 10.73 \\
\hline & ELZ14_27635 (sacB) & EC 2.4.1.10; glycoside hydrolase 68 family protein (levansucrase) & 6.33 \\
\hline \multirow{10}{*}{ Detoxification } & ELZ14_00175 (katE) & EC 1.11.1.6; catalase HPII & 8.02 \\
\hline & ELZ14_03625 (copA) & EC 3.6.3.54; copper-exporting P-type ATPase A & 3.70 \\
\hline & ELZ14_03915 (katG) & EC 1.11.1.21; catalase/peroxidase HPI & 6.31 \\
\hline & ELZ14_09480 (-) & EC 3.6.3.54; cadmium-translocating P-type ATPase & 5.50 \\
\hline & ELZ14_24395 (copA) & n.a.; copper resistance system multicopper oxidase & 2.25 \\
\hline & ELZ14_24400 (copB) & n.a.; copper resistance protein B & 1.40 \\
\hline & ELZ14_24405 (copC) & n.a.; Copper resistance protein $C$ & 3.03 \\
\hline & ELZ14_24410 (copD) & n.a.; Copper resistance D family protein & 2.07 \\
\hline & ELZ14_24510 (dps2) & EC 1.16.-.; DNA starvation/stationary phase protection protein & 9.59 \\
\hline & ELZ14_27055 (katB) & EC 1.11.1.6; catalase & 2.63 \\
\hline Lipopolysaccharides & ELZ14_31170 (-) & n.a.; lipoteichoic acid (LTA) synthase family protein & 1.48 \\
\hline
\end{tabular}




\subsubsection{Secondary Metabolism and Antibiotics}

Using antiSMASH, nine regions predicted to encode secondary metabolite synthesis genes were detected in the 3Re2-7 genome. These include three antiSMASH type 'NRPS' regions (nonribosomal peptide synthetase, region sM_r4, sM_r7 and sM_r9) which are described in Subsection '2.4.3. Pathogen inhibition'.

Region sM_r1 of the type 'NRPSfragment' (cluster with similarity to a NRPS fragment) showed a high similarity to the so-called Mangotoxin biosynthetic gene region (mgo-operon) that has been found in both pathogenic as well as beneficial Pseudomonas species [55,56]. Most probably its product acts as a regulator of different functions or as a Pseudomonas-specific switch for secondary metabolite production, rather than a toxin, but further details remain to be explored [55,56]. Moreover, no pathogenicity factor or compound has been linked to P. brassicacearum 3Re2-7 and the absence of any virulence, resistance and pathogen-associated genes was confirmed by means of Genomic IslandViewer 4 [30]. The predicted gene region sM_r1 comprises 28 genes. The core biosynthetic gene most probably is ELZ14_01120, encoding an amino acid adenylation domain-containing protein. ELZ14_01145 was predicted to be an additional biosynthetic gene. Its gene product carries an EAL domain which is named after its conserved residues and is found in diverse bacterial signalling proteins [51]. Further, the region involves several transport-related and regulatory genes.

A significant similarity to the aryl polyene biosynthetic gene cluster from the $\gamma$-proteobacterium Vibrio fischeri (BGC0000837, 40\% of genes show similarity) was reported for region sM_r2. An important role in Gram-negative cell biology is thought to be played by the products of gene clusters from the large and unexplored aryl polyene biosynthetic gene cluster family [57].

While antiSMASH predicted one region of type 'Bacteriocin' (region sM_r3), a screening with BAGEL4 yielded four putative bacteriocin gene clusters featuring similarity to genes coding for the synthesis of a microcin and three class III bacteriocins (colicin). Region sM_r3 does not show significant similarities to known clusters but one of the predicted core biosynthetic genes of the bacteriocin region sM_r3 detected by antiSMASH encodes a DUF629 domain-containing protein (ELZ14_06450). This domain of yet unknown function has been observed in several plant proteins. Therefore, this region is an interesting target for mutant analyses in combination with plant interaction studies.

By similarity to the beta-lactone fengycin biosynthesis cluster, region sM_r5 was predicted to represent the type 'betalactone'. Fengycin is known to be involved in biocontrol activity of Bacillus strains [58].

Further, the type III polyketide synthase (PKS) region (region sM_r6) predicted by antiSMASH is identical to the 2,4-diacetylphloroglucinol (2,4-DAPG) biosynthetic gene cluster from Pseudomonas fluorescens. Thereby, all genes required for the synthesis of the antibiotic 2,4-DAPG are present (Table 5).

The region assigned to the type 'Butyrolactone' (region sM_r8) showed no significant hits to known secondary metabolite clusters. By means of further detailed analysis, the gene operon needed for the production of the secondary metabolite hydrogen cyanide ( $h c n A B C$ ) was annotated.

A further determinant for production of secondary metabolites is acetohydroxyacid synthase (AHAS, EC 2.2.1.6). AHAS is the key enzyme in branched chain amino acid biosynthesis in bacteria. In the 3Re2-7 genome, the genes ilvH (ELZ14_26510) and ilvI (ELZ14_26515) coding for the small and large subunit of AHAS, respectively, were predicted. Also, the gene ivlC (ELZ14_26505) was annotated, coding for ketol-acid reductoisomerase (KARI, EC 1.1.1.86). Since AHAS and KARI are able to form precursors for a number of secondary metabolites such as cyanogenic glycosides, glucosinolates and acyl-sugars, both enzymes belong to the KEGG pathways 'Biosynthesis of secondary metabolites' (ec01110) and 'Biosynthesis of antibiotics' (ec01130) [50].

\subsubsection{Induced Systemic Resistance and Plant Growth Promotion}

P. brassicacearum 3Re2-7 has the metabolic potential to produce proteins involved in the synthesis of volatiles. Amongst others, bacterial volatiles such as 2,3-butanediol have been linked to Induced Systemic Resistance (ISR). In the rhizosphere, volatiles elicit root exudates which selectively affect 
different bacterial species [59,60]. Also, 2,3-butanediol has been shown to play a role as a bacterial protectant. It protects bacterial cells against putative harmful plant root exudates and low $\mathrm{pH}$ [61]. The translated protein sequence of the gene ELZ14_17085 shows 100\% identity to the Pseudomonas multispecies protein sequence of butanediol dehydrogenase. This enzyme is involved in the production of the volatile 2,3-butanediol [61].

Genetic determinants possibly involved in plant growth promotion include the acdS gene coding for 1-aminocyclopropane-1-carboxylate (ACC) deaminase (ELZ14_11285). ACC is the immediate precursor of the phytohormone ethylene. Through deamination, ACC gets broken down to ammonia and $\alpha$-ketobutyrate, which can be further metabolized by bacteria. At the same time, ACC deaminase lowers plant ethylene levels. This mechanism has been observed in plant growth promoting bacteria to facilitate plant growth. Also, the acdS gene product has been shown to have a positive impact on plant growth under different environmental stresses [62]. Pairwise amino acid sequence comparison of the predicted protein sequence of the acdS gene (ELZ14_11285) in the 3Re2-7 genome revealed a complete match to the aminocyclopropane-1-carboxylate deaminase/D-cysteine desulfhydrase family protein sequence of different Pseudomonas species, suggesting that the putative acdS gene in the 3Re2-7 genome is functional.

P. brassicacearum 3Re2-7 further encodes riboflavin synthase (EC 2.5.1.9, ELZ14_27880), which catalyzes the final reaction of riboflavin biosynthesis. Riboflavin stimulates plant growth and is known to function as protectant/elicitor of plant defense [63,64].

A region encoding a Type III secretion system (T3SS) was predicted (ELZ14_29730-29880). T3SSs are found in Gram-negative bacterial pathogens and symbionts and enable bacteria to inject effector proteins into the host cell [65]. Further, T3SSs are believed to have a significant role in the biology of plant growth promoting rhizobacteria such as P. fluorescens SBW25 [66].

Many plant-beneficial soil bacteria are capable of solubilizing phosphorus by synthesizing organic acids and acid phosphatases, making it available to plants [67]. Screening for genes related to phosphate solubilization uncovered a phytase gene (ELZ14_15680) and several unspecific phosphatase genes (listed in Table 6). Moreover, strain 3Re2-7 is most probably able to solubilize inorganic phosphate, since in its genome the $g c d$ gene coding for a glucose/quinate/shikimate family membrane-bound pyrroloquinoline quinone (PQQ)-dependent dehydrogenase was predicted (ELZ14_24970). This enzyme together with its cofactor PQQ catalyzes the production of gluconic acid [68]. All six genes of the operon for the pyrroloquinoline quinone coenzyme biosynthesis were observed, including the $p q q C$ gene coding for the PQQ synthase ( $\left.p q q A B C D E F, E L Z 14 \_28615-28640\right)$. The presence of a phytase and several phosphatases suggests that $P$. brassicacearum 3Re2-7 is able to solubilize high amounts of phosphorus, which might add to its plant beneficial potential.

\subsubsection{Pathogen Inhibition}

The biosynthetic pathway for the production of aromatic amino acids majorly contributes to the broad functional spectrum of organisms in nature. Various pigments, siderophores, signaling compounds, defense metabolites, structural compounds, antibiotics and other secondary metabolites are derived from this pathway [69]. In Pseudomonas species, this pathway starts with chorismate [69]. Chorismate can be transformed into anthranilate, by the action of anthranilate synthase (TrpE/TrpG), which is encoded in the genome (ELZ14_28410/ELZ14_28400). Anthranilate synthase is an enzyme of the menaquinone, siderophore and tryptophan (MST) class [70]. Siderophores can enhance iron acquisition and suppress target pathogens in the rhizosphere through iron competition and are therefore of importance for biocontrol. The $p c h D C B A$ operon is required for the biosynthesis of the high-affinity siderophore salicylate, which is also an intermediate in the biosynthetic pathway of pyochelin, another siderophore [71]. Of the $p c h D C B A$ operon, the gene coding for isochorismate lyase PchB (ELZ14_13330 pchB) was predicted in the genome of P. brassicacearum 3Re2-7. Also, the neigboring gene is annotated as isochorismatase family protein (ELZ14_13335), which is involved in the 
biosynthesis of several siderophores, such as 2,3-dihydroxybenzoylglycine, enterobactin, bacillibactin and vibriobactin [72].

For the antiSMASH Nonribosomal Polyketide Synthase (NRPS)-type regions sM_r4, sM_r7 and sM_r9 (Figure 2), significant similarities to known pyoverdine, serobactins, cupriachelin, taiwachelin, bleomycin, viscosin and delftibactin biosynthetic gene regions were reported. Region sM_r9 encoding an unknown non-ribosomal polyketide synthetase, harbors the pvdA gene (ELZ14_21210). Its gene product ornithine 5-monooxygenase catalyzes the first step in the biosynthesis of all hydroxamate-containing siderophores, such as pyoverdine. It further includes the putative core biosynthetic genes predicted to code for three non-ribosomal peptide synthases (ELZ14_21260, ELZ14_21285 and ELZ14_21295), an amino acid adenylation domain-containing protein (ELZ14_21290) and a peptide synthase (ELZ14_21300). Also, transport related genes (ELZ14_21220-21230, ELZ14_21255 and ELZ14_21280) were annotated in this region, suggesting an export mechanism. Further, genes of the cluster show similarity to genes of a pyoverdine NRPS gene cluster from P. protegens (BGC0000413). These results suggest that this region encodes an unknown NRPS and that its product might be a siderophore. Detailed analyses of the modular architectures of PKS/NRPS clusters often enable the prediction of the produced compounds, even if the biological function or bioactivity remains unknown [8]. The predicted core structure of the compound produced by the NRPS is shown in Supplementary File S1. Its predicted structure resembles the antibiotic peptide phosphinothricin tripeptide (NOR00670) listed in the Norine database [73].

Region sM_r4 of the regions identified by antiSMASH also includes a core biosynthetic gene whose gene product was annotated as non-ribosomal peptide synthetase (ELZ14_09165). The most similar known cluster is also the P. protegens pyoverdine biosynthetic gene cluster BGC0000413.

The third predicted NRPS type cluster located in region sM_r7, has five annotated core biosynthetic genes, predicted to encode four non-ribosomal peptide synthetases (ELZ14_12310-12325) and a peptide synthase (ELZ14_12335). Transport-related genes are also present in this region (ELZ14_12285, ELZ14_12305 and ELZ14_12345). The cluster is most similar to the known cupriachelin cluster from Ralstonia eutropha (BGC0000330) and the predicted product has similarity to the peptide chrysobactin (NOR00210), which shows siderophore activity.

On that basis, it can be hypothesized that $P$. brassicacearum 3 Re2-7 is able to produce siderophores.

\subsubsection{Other Biocontrol Features}

Exopolysaccharides are known to aid colonization and biofilm persistence, which is especially important in harsh and competitive environments like soil and rhizosphere [74]. The P. brassicacearum 3Re2-7 chromosome contains a cluster comprising twelve genes (ELZ14_25290-350) predicted to encode functions related to the biosynthesis of the exopolysaccharide alginate. Further, it may have the genetic potential to produce the exopolysaccharide levan, since it was predicted to encode a glycosyl hydrolase 68 (GH68) family protein (ELZ14_27635). The GH68 family includes several bacterial levansucrase enzymes.

P. brassicacearum 3Re2-7 encodes genes for the biosynthesis of various bacterial cell envelope components, that is, lipopolysaccharides (LPS) (ELZ14_02690, ELZ14_02695, ELZ14_02715, ELZ14_04750, ELZ14_04755, ELZ14_04760, ELZ14_20190). LPS can serve as a barrier to many antibiotics and changes in lipid composition may enable microorganisms to maintain outer membrane functions while facing environmental fluctuations. Interestingly, P. brassicacearum 3Re2-7 encodes a LTA synthase family protein predicted to be involved in the biosynthesis of lipoteichoic acid (ELZ14_31170). This is atypical for Gram-negative bacteria. In Gram-positive bacteria, teichoic acid is believed to i.a. function in biofilm formation and host tissue adhesion [75]. Most probably, the production of lipoteichoic acid adds to the rhizosphere competence of strain 3Re2-7.

Gene ELZ14_17635 is a homolog of $a c n B$, coding for aconitase B. The production of aconitase B has been shown to be required for optimal growth sustainment of pathogenic Xanthomonas campestris in pepper plants. Since P. brassicacearum 3Re2-7 is a plant growth promoting bacterium, this trait most 
probably indicates a mechanism to enhance competitiveness in the rhizosphere or endorhiza. Also, two mqo-homologous genes (ELZ14_13780, ELZ14_26110) coding for malate:quinone oxidoreductase were identified. This enzyme has been hypothesized to be involved in growth adaptation to the host environment in pathogenic P. syringae [76]. In the beneficial P. brassicacearum, it might be advantageous for the endophytic lifestyle. Further putative biocontrol determinants are listed in Table 8.

P. brassicacearum 3Re2-7 encodes the potential to synthesize a great variety of biocontrol related compounds, including secondary metabolites, bacteriocins and siderophores. Summarized, corresponding genetic determinants detected by genome mining make P. brassicacearum 3Re2-7 a promising biocontrol agent.

\subsection{Transcriptome Sequencing of P. brassicacearum 3Re2-7 Confirms Expression of Biocontrol Related Genes}

In order to verify transcription of predicted biocontrol-related genes and to detect genes organized in operons, whole-transcriptome sequencing was applied. Transcriptomic analyses in the context of biocontrol properties often implement RNA-Seq to reveal responses of a BCA to a pathogen $[15,77,78]$. Phenotypic variation of Pseudomonas species has been elucidated as relevant in competitive root colonization and biological control of phytopathogens $[79,80]$. The production of exo-enzymes and secondary metabolites in phase variation has been shown to affect colonization behaviour and biocontrol activity of rhizosphere bacteria [80]. Therefore, under laboratory conditions, some genes may be silent due to the absence of appropriate triggers. Since these could be activated by, for example, co-cultivation, we included transcriptomic data from an co-cultivation of P. brassicacearum 3Re2-7 with the pythopathogenic fungus Rhizoctonia solani AG1-IB. Transcriptome sequencing of P. brassicacearum yielded in total 46,076,764 reads amounting to 3,428,193,102 bases. With BBMap, 40,328,306 assignable mappings were generated. Feature coverage analysis revealed, that 4983 of the 6267 predicted genes are covered considering a minimum covered percentage of $90 \%$ and a minimum count of ten reads per gene. 6113 genes were considered as transcribed, since their Transcript Per Million (TPM) count was above the determined cutoff (TPM $>1)$. No genes were reported as differentially expressed ( $p$-adjusted value $<0.05$ and $\log 2$ fold change $>2$ ). A possible explanation might be, that the biocontrol features making P. brassicacearum 3Re2-7 effective against $R$. solani are constitutively expressed as it has been observed for biocontrol features of different Bacillus or Trichoderma biocontrol strains [81-83]. A recent study elaborates the transcriptional response of the biocontrol strain $P$. fluorescens In 5 to $R$. solani. The authors found no significant changes in global gene expression but noticed that genes possibly involved in metabolite detoxification are highly upregulated [15]. Based on the results obtained in this study, we evaluated the P. brassicacearum 3Re2-7 transcriptional profiles of both treatments in a combined manner (see Tables 5 to 8). 780 genes were determined as highly transcribed, of these 68 as exceptionally highly transcribed, based on the log- 2 transformed TPM distribution (Figure 5 and Figure S2).

Among the exceptionally highly transcribed genes is ELZ14_28620, encoding the pyrroloquinoline quinone precursor peptide PqqA. PQQ, a bacterial redox active cofactor for numerous alcohol and aldose dehydrogenases, is derived from the two amino acids glutamate and tyrosine encoded in the precursor peptide PqqA [84]. Moreover, as before-mentioned, it is an important cofactor in inorganic phosphate solubilization and is associated with antifungal activity and induction of systemic resistance [85]. The $p q q A$ gene is part of the $p q q A B C D E F$ operon mentioned before. The other genes of this operon were also transcribed but at moderate rates (log2-transformed TPMs of 3.98 to 6.8).

The four genes ELZ14_06515-30 are all among the top 68 highly expressed genes. They include the three genes encoding structural enzymes of the arginine deiminase (ADI) pathway: carbamate kinase, ornithine carbamoyltransferase and arginine deiminase. In prokaryotes, the biosynthesis of the amino acid arginine, plays a significant role in nitrogen metabolism [86]. Also, arginine and its precursors are involved in the biosynthesis of several metabolites such as polyamines and some antibiotics [87].

Interestingly, four genes of the phlABCEDFGH cluster ( $p h l D, C, A$ and $G$ ) needed for the production of the antibiotic 2,4-DAPG are among the 68 exceptionally highly transcribed genes. 


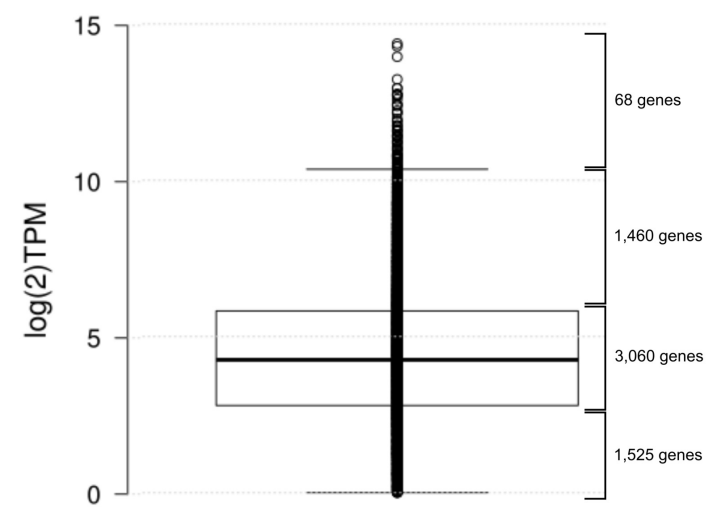

Figure 5. Box-Whisker plot of the log2-transformed Transcript per Million (TPM) values of transcribed genes. The center line shows the median (4.25), box limits indicate the 25th and 75th percentiles as determined by Tukey ( 2.79 and 5.82, respectively). Whiskers extend up to 1.5 times the interquartile range from the 25 th and 75 th percentiles ( 0.00 and 10.35 , respectively), data points are plotted as open circles, $\mathrm{n}=6113$ sample points.

Further, among the top 68 are ELZ14_09515-30 and ELZ14_09515, which encode subunits of cbb3-type cytochrome-c oxidase. Cytochrome cbb3 oxidases are found almost exclusively in Proteobacteria and represent a distinctive class of proton-pumping respiratory heme-copper oxidases (HCO) which are expressed only under microaerobic conditions and thought to be required for colonisation of anoxic tissues [88].

Operon detection applying the ReadXplorer software [89] yielded 1,161 operons (co-transcribed sets of genes resulting in a single polycistronic messenger RNA). Genes were regarded to constitute an operon if a minimum of five spanning reads in sense direction connected the genes. The largest operon harboured 20 genes (ELZ_02445-02540) coding for inter alia enzymes that are associated with fatty acid and lipid biosynthesis and translocation of lipoproteins from the inner membrane to the outer membrane. The operon also includes an aromatic amino acid lyase gene that may be involved in the biosynthesis of a wide variety of secondary metabolites such as flavonoids, furanocoumarin phytoalexins and cell wall components [51].

The $h c n A B C$ cluster, which is one of the named biocontrol determinants (Table 5), also was reported as operon. Further, it was shown to be stongly transcribed (log2-transformed TPM $>9$ ).

Of the phlABCDEFGH cluster (Table 5), the phlABC operon was reported. The phlABC operon encodes a three-protein complex to catalyze the first step of 2,4-DAPG biosynthesis [90]. PhlABC and phlD show high transcription ( $\log 2$-transformed TPM $>10$ ).

Interestingly, the aco $A B C$ genes (Table 6) that encode the subunits of acetoin dehydrogenase were reported to be transcribed in an operon including a 2,3-butanediol dehydrogenase (ELZ14_17085) and an ATP-NAD kinase gene (ELZ14_17105). NAD and NADH are cofactors of 2,3-butanediol dehydrogenase [91]. 2,3-Butanediol dehydrogenase was shown to be able to catalyze the irreversible reduction of diacetyl to (S)-acetoin in Corynebacterium glutamicum. Acetoin (3-hydroxy-2-butanone) is a ketone, which is known to be produced by several PGPRs and has been shown to promote plant growth [92]. The transcription rate of this operon is moderate (log2-transformed TPM of 4.5).

The riboflavin biosynthetic pathway (RBP) genes are clustered in operons in most bacteria but their genetic organization varies among species $[93,94]$. In P. brassicacearum 3Re2-7, next to a single ribBA locus and a single ribF locus, a cluster of RBP genes in the order ribFAHBED is present (Table 6). Of these, operon detection reported $r i b B$, ribE and $r i b D$ to be transcribed in an operon with a transcriptional regulator NrdR (ELZ14_27890). The association of bacterial rib genes with this transcriptional regulator has been observed previously [94]. 
Some biocontrol determinants of P. brassicacearum 3Re2-7 could be produced constitutively. This might be advantageous to sustain in competitive environments like soil and endorhiza. The presence of multiple potentially antifungal gene products points to a synergistic mode of action, as it was shown for Bacillus amyloliquefaciens FZB42 [8]. Therefore, the construction of multigenetic mutants in combination with metabolomic analyses could aid the understanding of cooperativity regarding biocontrol traits.

\section{Materials and Methods}

\subsection{PacBio Library Preparation, Sequencing and Genome Assembly}

Genomic DNA was purified from P. brassicacearum 3Re2-7 (kindly provided by Dr. Rita Grosch, IGZ Großbeeren, Germany) grown in LB medium to the late-logarithmic growth phase by applying the Gentra Puregene Yeast/Bacteria Kit as outlined in the manual provided by the supplier (Qiagen, Hilden, Germany). The extracted DNA further was teated with the Zymo Genomic DNA Clean \& Concentrator ${ }^{T M}-10$ kit. SMRTbell ${ }^{T M}$ template library was prepared according to the instructions from PacificBiosciences (Menlo Park, CA, USA) following the 'Procedure \& Checklist - Greater Than $10 \mathrm{~kb}$ Template Preparation'. Briefly, for preparation of $15 \mathrm{~kb}$ libraries, $8 \mu \mathrm{g}$ genomic DNA was sheared using g-tubes ${ }^{T M}$ (Covaris, Woburn, MA, USA) according to the manufacturer's instructions. DNA was end-repaired and ligated overnight to hairpin adapters applying components from the DNA/Polymerase Binding Kit P6 from Pacific Biosciences (Menlo Park, CA, USA). Reactions were carried out according to the instructions of the manufacturer. BluePippin ${ }^{T M}$ Size-Selection to greater than $4 \mathrm{~kb}$ was performed according to the manufacturer's instructions (Sage Science, Beverly, MA, USA). Conditions for annealing of sequencing primers and binding of polymerase to purified SMRTbell ${ }^{T M}$ template were assessed with the Calculator in RS Remote (PacificBiosciences, Menlo Park, CA, USA). SMRT sequencing was carried out on the PacBio RSII (PacificBiosciences, Menlo Park, CA, USA) taking one 240-min movie for one SMRT cell using the P6 Chemistry. Sequencing resulted in 90,452 post-filtered reads with a mean read length of $9920 \mathrm{bp}$. SMRT Cell data was assembled using the "RS_HGAP_Assembly.3" protocol included in SMRT Portal version 2.3.0 using default parameters. The assembly revealed a circular chromosome. Validity of the assembly was checked using the "RS_Bridgemapper.1" protocol. The chromosome was circularized, particularly artificial redundancies at the ends of the contigs were removed and adjusted to $d n a A$ as the first gene. Error-correction was performed by a mapping of 1.5 Million paired-end MiSeq Illumina reads of $2 \times 300 \mathrm{bp}$ onto the finished genome using BWA [95] with subsequent variant and consensus calling using VarScan [96]. A consensus concordance of QV60 could be confirmed for the genome. The genome sequence was deposited in NCBI GenBank under Accession Number CP034725.

\subsection{Genome Annotation and Mining}

Annotation was carried out using the NCBI Prokaryotic Genome Annotation Pipeline (PGAP) [97]. PHASTER [28] was used to identify prophage sequences, ResFinder-3.1 [29] to detect antimicrobial resistance genes. Genomic Islands (GIs) were detected and visualized in IslandViewer 4 [30]. A screening for bacteriocins was performed applying BAGEL4 [98] (http://bagel4.molgenrug.nl/). For the identification of secondary metabolites clusters antiSMASH 5 beta was used [99]. Predicted NRPS products were searched in the NORINE database [100].

\subsection{Comparative Analysis}

All publicly available genomes of Pseudomonas brassicacearum strains reported before June 2019 (Table 1) were obtained in GenBank format from the NCBI genome database (https://www.ncbi. nlm.nih.gov/genome). P. kilonensis strains were also included, since this species has previously been suggested as the 'junior synonym' to P. brassicacearum [35]. Metadata of the strains was derived from the associated publications if available (Table 1). Further, genomes of type strains of the genus Pseudomonas 
as named in the list of prokaryotic names with standing in nomenclature (http:/ / www.bacterio.net) were obtained from the NCBI genome database in GenBank format. Namely, the type strain of the genus, P. aeruginosa DSM 50071 ${ }^{T}$ [101] and several type strains belonging to the Pseudomonas fluorescens group (P. fluorescens DSM 50090 ${ }^{T}$ [101], P. veronii DSM 11331 ${ }^{T}$ [102], P. azotoformans NBRC 12693 ${ }^{T}$ [101], P. trivialis DSM $14937^{T}$ [103], P. orientalis DSM 17489 ${ }^{T}$ [104] and P. synxantha NBRC 3913 ${ }^{T}$ [101]) were selected for phylogenetic analysis. Accession numbers of all used strains are listed in Supplementary Table S1. The annotated genomes in GenBank format were used for core genome calculation, construction of a phylogenetic tree, singleton gene analysis and calculation of Average Amino-acid Identity as implemented in EDGAR 2.0 [42]. For calculation of a phylogenetic tree, genomes of known biocontrol strains were integrated. The tree including 33 genomes was built on basis of 2331 core genes per genome. Core genes were aligned using MUSCLE [105] and concatenated to one alignment. FastTree software (http:/ / www.microbesonline.org/fasttree/) was used to generate an approximatelymaximum-likelihood phylogenetic tree. All the nodes have bootstrap values of 100 . The phylogenetic tree constructed in EDGAR 2.0 was exported in newick format and visualized using Evolview v2 [106]. The DDH estimates were calculated using the Genome-To-Genome Distance Calculator [107] chosing the recommended formula 2. BRIG [31] was used for visualizing the comparison of a reference sequence to a set of query sequences.

\subsection{Transcriptome Analysis}

Cultures were grown on Waksman agar containing $5 \mathrm{~g} / \mathrm{L}$ of Peptone (Roth, Karlsruhe, Germany), 10 g/L glucose (VWR, Darmstadt, Germany), 3 g/L meat extract (Roth), 5 g/L NaCl (Oxoid, Basingstoke, UK), $20 \mathrm{~g} / \mathrm{L}$ Agar-Agar Kobe I (Roth) and distilled water (to $1 \mathrm{~L}$ ), pH 6,8. The strain 3Re2-7 was grown without and with confrontation with Rhizoctonia solani AG1-IB isolate 7/3/14 [108]. Each treatment included four replicates which were used to determine the Pseudomonas brassicacearum 3Re2-7 transcriptome. After incubation in the dark at room temperature for $36 \mathrm{~h}$, samples of bacterial material were stored in RNAprotect bacterial reagent (Qiagen, Valencia, CA, USA). RNA extraction was done using the RNeasy Mini Kit (Qiagen, Valencia, CA, USA). RNA quality was assessed using the Agilent 2100 bioanalyzer (Agilent Technologies, Palo Alto, CA, USA). The cDNA library generation was performed according to Pátek et al., 2013 [109]. For sequencing, advisements for RNA-Seq profiling of bacterial transcriptomes as stated in Haas et al., 2012 [110] were considered. The cDNA library was sequenced using TruSeq stranded mRNA on the Illumina MiSeq system generating $2 \times 75$ bp paired-end reads. Obtained RNA-Seq reads were adapter and quality trimmed by means of BBDuk (Bushnell, http:/ /jgi.doe.gov/data-and-tools/bbtools/). Fastx_reverse_complement from FASTX-Toolkit (http: / / hannonlab.cshl.edu/fastx_toolkit/) was used to maintain the sense direction of transcripts. Then the reads were mapped onto the P. brassicacearum 3Re2-7 genome using BBMap [111]. Transcriptome analysis was done with ReadXplorer 2 [89], for calculation of read counts and Transcript Per Million (TPM) values, for operon detection and feature coverage analysis the 'Single Perfect Match' and 'Single Best Match' options were used. The transcription profiles from both treatments were combined for analysis, since differential gene expression analysis with DESeq2 [112] using the cutoffs p-adjusted value $<0.05$ and $\log 2$ fold change $>2$ yielded no differentially expressed genes. Genes were presumed non-transcribed if the log2-transformed TPM value was below zero, all genes with positive values were considered as verified. Based on the distribution of log2-transformed TPM-values (Figure 5 and Figure S2), genes were assumed highly transcribed, if their log2-transformed TPM value was greater than seven (corresponding non-transformed TPM > 128). Finally, operon detection was performed with a minimum of five spanning reads. 


\section{Conclusions}

The strain P. brassicacearum 3Re2-7 was selected for genome sequencing due to its biocontrol and plant growth promoting properties [24-27,32]. Additionally, the strain is commercially applied as plant growth promoting biostimulant. We established its genome sequence as a basis for genomemining, comparative genomics and transcriptional profiling. We detected putative genetic biocontrol determinants, as well as potentially new biocontrol related genes and gene clusters by genome-mining. Based on the now known genome sequence, the predicted functions of the identified genes and transcripts can be investigated by, for example, mutagenesis experiments. Through comparative analysis, we found unique gene clusters which represent interesting targets for mutagenesis experiments in order to elucidate their function. Our data demonstrated that in P. brassicacearum 3Re2-7 biocontrol genes including genes involved in secondary metabolite production are transcribed. These findings underpin its potential as microbial biocontrol agent. Therefore, this study contributes to the basic research towards a safe use of indigenous as well as inoculated Pseudomonas brassicacearum biocontrol agents for a sustainable disease management in agriculture.

Supplementary Materials: The following are available online at http:/ /www.mdpi.com/2073-4425/10/8/601/s1. Figure S1: By antiSMASH predicted core structure of the putative NRPS encoded by sM_r9. Figure S2: Distribution of log2-transformed Transcript Per Million (TPM) values of all genes. Table S1: Accession numbers of all strains used in bioinformatic analyses.

Author Contributions: conceptualization, J.N.; methodology, J.N., G.T.T. and J.W.; software, J.N. and T.W.L.; validation, J.N., G.T.T., B.V. and J.W.; formal analysis, J.N.; investigation, J.N.; resources, J.N., R.G. and A.S.; data curation, J.N., B.B.; writing—original draft preparation, J.N.; writing—review and editing, J.N., G.T.T., J.H., T.W.L., J.W., B.V., A.W., B.B., C.S., J.O., R.G., A.P. and A.S.; visualization, J.N., J.H. and T.W.L.; supervision, A.S. and A.P.; project administration, A.S. and A.P.; funding acquisition, A.S. and R.G.

Funding: This work was funded by the German Federal Ministry of Education and Research (BMBF) in the frame of the BonaRes program, grant number 22016308. The authors also acknowledge support by the Projektträger Jülich (PtJ).

Acknowledgments: A.S. acknowledges funding from the European Union's Horizon 2020 research and innovation programme under grant agreement No. 818431 (SIMBA). This output reflects only the author's view and the Research Executive Agency (REA) cannot be held responsible for any use that may be made of the information contained therein. J.N. and J.H. acknowledge the German Federal Ministery of Food and Agriculture (BMEL) for financial support via the Fachagentur für Nachwachsende Rohstoffe e.V. (FNR) of the research project BMP-III (FKZ 22404015). The great support of the EDGAR project by Jochen Blom is acknowledged. For excellent technical assistance regarding PacBio genome sequencing Simone Severitt and Nicole Heyer are acknowledged. Support was also provided by the German Network for Bioinformatics Infrastructure (de.NBI).

Conflicts of Interest: The authors declare no conflict of interest. The funders had no role in the design of the study, in the collection, analyses, or interpretation of data, in the writing of the manuscript, or in the decision to publish the results.

\section{References}

1. Hartmann, M.; Frey, B.; Mayer, J.; Mäder, P.; Widmer, F. Distinct soil microbial diversity under long-term organic and conventional farming. ISME J. 2015, 9, 1177-1194. [CrossRef] [PubMed]

2. Oerke, E.C. Crop Losses to Pests J. Agric. Sci. 2006, 144, 31-43. [CrossRef]

3. Berg, G.; Köberl, M.; Rybakova, D.; Müller, H.; Grosch, R.; Smalla, K. Plant Microbial Diversity Is Suggested as the Key to Future Biocontrol and Health Trends. FEMS Microbiol Ecol. 2017, 93, doi:10.1093/femsec/fix050. [CrossRef] [PubMed]

4. Chowdhury, S.P.; Dietel, K.; Rändler, M.; Schmid, M.; Junge, H.; Borriss, R.; Hartmann, A.; Grosch, R. Effects of Bacillus amyloliquefaciens FZB42 on Lettuce Growth and Health under Pathogen Pressure and Its Impact on the Rhizosphere Bacterial Community. PLoS ONE 2013, 8, e68818. doi:10.1371/journal.pone.0068818. [CrossRef] [PubMed]

5. Garrido-Sanz, D.; Meier-Kolthoff, J.P.; Göker, M.; Martín, M.; Rivilla, R.; Redondo-Nieto, M. Genomic and genetic diversity within the Pseudomonas fluoresces complex. PLOS ONE 2016, 11, e0150183.

6. $\quad$ Pieterse, C.M.; Zamioudis, C.; Berendsen, R.L.; Weller, D.M.; Van Wees, S.C.; Bakker, P.A. Induced Systemic Resistance by Beneficial Microbes. Annu. Rev. Phytopathol. 2014, 52, 347-375. [CrossRef] [PubMed] 
7. Maksimov, I.V.; Abizgil'dina, R.R.; Pusenkova, L.I. Plant growth promoting rhizobacteria as alternative to chemical crop protectors from pathogens (review). Appl. Biochem. Microbiol. 2011, 47, 333-345. [CrossRef]

8. Paterson, J.; Jahanshah, G.; Li, Y.; Wang, Q.; Mehnaz, S.; Gross, H. The contribution of genome mining strategies to the understanding of active principles of PGPR strains. FEMS Microbiol. Ecol. 2017, 93, fiw249. [CrossRef]

9. Nelkner, J.; Henke, C.; Lin, T.W.; Pätzold, W.; Hassa, J.; Jaenicke, S.; Grosch, R.; Pühler, A.; Sczyrba, A.; Schlüter, A. Effect of Long-Term Farming Practices on Agricultural Soil Microbiome Members Represented by Metagenomically Assembled Genomes (MAGs) and Their Predicted Plant-Beneficial Genes. Genes 2019, 10, 424. [CrossRef]

10. Mishra, J.; Arora, N.K. Secondary metabolites of fluorescent pseudomonads in biocontrol of phytopathogens for sustainable agriculture. Appl. Soil Ecol. 2018, 125, 35-45. [CrossRef]

11. Couillerot, O.; Prigent-Combaret, C.; Caballero-Mellado, J.; Moënne-Loccoz, Y. Pseudomonas fluorescens and closely-related fluorescent pseudomonads as biocontrol agents of soil-borne phytopathogens. Lett. Appl. Microbiol. 2009, 48, 505-512. [CrossRef] [PubMed]

12. Barret, M.; Frey-Klett, P.; Boutin, M.; Guillerm-Erckelboudt, A.Y.; Martin, F.; Guillot, L.; Sarniguet, A. The plant pathogenic fungus Gaeumannomyces graminis var. tritici improves bacterial growth and triggers early gene regulations in the biocontrol strain Pseudomonas fluorescens Pf29Arp. New Phytol. 2009, 181, 435-447. [CrossRef] [PubMed]

13. Hernández-León, R.; Rojas-Solís, D.; Contreras-Pérez, M.; Orozco-Mosqueda, M.d.C.; Macías-Rodríguez, L.I.; Reyes-de la Cruz, H.; Valencia-Cantero, E.; Santoyo, G. Characterization of the antifungal and plant growth-promoting effects of diffusible and volatile organic compounds produced by Pseudomonas fluorescens strains. Biol. Control 2015, 81, 83-92. [CrossRef]

14. Michelsen, C.F.; Watrous, J.; Glaring, M.A.; Kersten, R.; Koyama, N.; Dorrestein, P.C.; Stougaard, P. Nonribosomal peptides, key biocontrol components for Pseudomonas fluorescens in5, isolated from a Greenlandic suppressive soil. mBio 2015, 6, e00079. [CrossRef] [PubMed]

15. Hennessy, R.C.; Glaring, M.A.; Olsson, S.; Stougaard, P. Transcriptomic profiling of microbe-microbe interactions reveals the specific response of the biocontrol strain P. fluorescens In5 to the phytopathogen Rhizoctonia solani. BMC Res. Notes 2017, 10, 376. [CrossRef] [PubMed]

16. Achouak, W.; Sutra, L.; Heulin, T.; Meyer, J.M.; Fromin, N.; Degraeve, S.; Christen, R.; Gardan, L. Pseudomonas brassicacearum sp. nov. and Pseudomonas thivervalensis sp. nov., two root-associated bacteria isolated from Brassica napus and Arabidopsis thaliana. Int. J. Syst. Evol. Microbiol. 2000, 50, 9-18. [CrossRef]

17. Zhou, T.; Chen, D.; Li, C.; Sun, Q.; Li, L.; Liu, F.; Shen, Q.; Shen, B. Isolation and characterization of Pseudomonas brassicacearum $\mathrm{J} 12$ as an antagonist against Ralstonia solanacearum and identification of its antimicrobial components. Microbiol. Res. 2012, 167, 388-394. [CrossRef]

18. Ortet, P.; Barakat, M.; Lalaouna, D.; Fochesato, S.; Barbe, V.; Vacherie, B.; Santaella, C.; Heulin, T.; Achouak, W. Complete genome sequence of a beneficial plant root-associated bacterium, Pseudomonas brassicacearum. J. Bacteriol. 2011, 193, 3146. [CrossRef]

19. Loewen, P.C.; Switala, J.; Fernando, W.G.D.; de Kievit, T. Genome Sequence of Pseudomonas brassicacearum DF41. Genome Announc. 2014, 2, 10-11. [CrossRef]

20. Khayi, S.; des Essarts, Y.R.; Mondy, S.; Moumni, M.; Hélias, V.; Beury-Cirou, A.; Faure, D. Draft Genome Sequences of the Three Pectobacterium-Antagonistic Bacteria Pseudomonas brassicacearum PP1-210F and PA1G7 and Bacillus simplex BA2H3. Genome Announc. 2015, 3, e01497-14. [CrossRef]

21. Zachow, C.; Müller, H.; Monk, J.; Berg, G. Complete genome sequence of Pseudomonas brassicacearum strain L13-6-12, a biological control agent from the rhizosphere of potato. Stand. Genom. Sci. 2017, 12, 6. doi:10.1186/s40793-016-0215-1. [CrossRef] [PubMed]

22. Novinscak, A.; Gadkar, V.J.; Joly, D.L.; Filion, M. Complete Genome Sequence of Pseudomonas brassicacearum LBUM300, a Disease-Suppressive Bacterium with Antagonistic Activity toward Fungal, Oomycete, and Bacterial Plant Pathogens. Genome Announc. 2016, 4, 2012-2013. [CrossRef] [PubMed]

23. Lanteigne, C.; Gadkar, V.J.; Wallon, T.; Novinscak, A.; Filion, M. Production of DAPG and HCN by Pseudomonas sp. LBUM300 contributes to the biological control of bacterial canker of tomato. Phytopathology 2012, 102, 967-973. [CrossRef] [PubMed]

24. Kai, M.; Effmert, U.; Berg, G.; Piechulla, B. Volatiles of bacterial antagonists inhibit mycelial growth of the plant pathogen Rhizoctonia solani. Arch. Microbiol. 2007, 187, 351-360. [CrossRef] [PubMed] 
25. Berg, G.; Krechel, A.; Ditz, M.; Sikora, R.A.; Ulrich, A.; Hallmann, J. Endophytic and ectophytic potatoassociated bacterial communities differ in structure and antagonistic function against plant pathogenic fungi. FEMS Microbiol. Ecol. 2005, 51, 215-229. [CrossRef] [PubMed]

26. Faltin, F.; Lottmann, J.; Grosch, R.; Berg, G. Strategy to select and assess antagonistic bacteria for biological control of Rhizoctonia solani Kühn. Can. J. Microbiol. 2004, 50, 811-820. [CrossRef] [PubMed]

27. Scherwinski, K.; Grosch, R.; Berg, G. Effect of bacterial antagonists on lettuce: Active biocontrol of Rhizoctonia solani and negligible, short-term effects on nontarget microorganisms. FEMS Microbiol. Ecol. 2008, 64, 106-116. [CrossRef] [PubMed]

28. Arndt, D.; Grant, J.R.; Marcu, A.; Sajed, T.; Pon, A.; Liang, Y.; Wishart, D.S. PHASTER: A better, faster version of the PHAST phage search tool. Nucleic Acids Res. 2016, 44, W16-W21. [CrossRef]

29. Zankari, E.; Hasman, H.; Cosentino, S.; Vestergaard, M.; Rasmussen, S.; Lund, O.; Aarestrup, F.M.; Larsen, M.V. Identification of acquired antimicrobial resistance genes. J. Antimicrob. Chemother. 2012, 67, 2640-2644. [CrossRef]

30. Bertelli, C.; Laird, M.R.; Williams, K.P.; Lau, B.Y.; Hoad, G.; Winsor, G.L.; Brinkman, F.S. IslandViewer 4: Expanded prediction of genomic islands for larger-scale datasets. Nucleic Acids Res. 2017, 45, W30-W35. [CrossRef]

31. Alikhan, N.F.; Petty, N.K.; Ben Zakour, N.L.; Beatson, S.A. BLAST Ring Image Generator (BRIG): simple prokaryote genome comparisons. BMC Genom. 2011, 12, 402. [CrossRef] [PubMed]

32. Krechel, A.; Faupel, A.; Hallmann, J.; Ulrich, A.; Berg, G. Potato-associated bacteria and their antagonistic potential towards plant-pathogenic fungi and the plant-parasitic nematode Meloidogyne incognita (Kofoid \& White) Chitwood. Can. J. Microbiol. 2002, 48, 772-786. [PubMed]

33. Madhaiyan, M.; Poonguzhali, S.; Saravanan, V.S.; Selvapravin, K.; Duraipandiyan, V.; Al-Dhabi, N.A. Pseudomonas sesami sp. nov., a plant growth-promoting Gammaproteobacteria isolated from the rhizosphere of Sesamum indicum L. Antonie van Leeuwenhoek 2017, 110, 843-852. [CrossRef] [PubMed]

34. Fournier, P.E.; Rossi-Tamisier, M.; Benamar, S.; Raoult, D. Cautionary tale of using 16S rRNA gene sequence similarity values in identification of human-associated bacterial species. Int. J. Syst. Evol. Microbiol. 2015, 65, 1929-1934.

35. Tran, P.N.; Savka, M.A.; Gan, H.M. In-silico taxonomic classification of 373 genomes reveals species misidentification and new genospecies within the genus Pseudomonas. Front. Microbiol. 2017, 8, 1296. [CrossRef] [PubMed]

36. Luo, C.; Rodriguez-R, L.M.; Konstantinidis, K.T. MyTaxa: An advanced taxonomic classifier for genomic and metagenomic sequences. Nucleic Acids Res. 2014, 42, e73. [CrossRef] [PubMed]

37. Rodriguez-R, L.M.; Konstantinidis, K.T. Bypassing Cultivation To Identify Bacterial Species. Microbe Mag. 2016, 9, 111-118. [CrossRef]

38. des Essarts, Y.R.; Cigna, J.; Quêtu-Laurent, A.; Caron, A.; Munier, E.; Beury-Cirou, A.; Hélias, V.; Faure, D. Biocontrol of the potato blackleg and soft rot diseases caused by Dickeya dianthicola. Appl. Environ. Microbiol. 2016, 82, 268-278. [CrossRef]

39. Keel, C.; Weller, D.M.; Natsch, A.; Défago, G.; Cook, R.J.; Thomashow, L.S. Conservation of the 2,4-diacetylphloroglucinol biosynthesis locus among fluorescent Pseudomonas strains from diverse geographic locations. Appl. Environ. Microbiol. 1996, 62, 552-563.

40. Eng, W.W.H.; Gan, H.M.; Gan, H.Y.; Hudson, A.O.; Savka, M.A. Whole-Genome Sequence and Annotation of Octopine-Utilizing Pseudomonas kilonensis (Previously P. fluorescens) Strain 1855-344. Genome Announc. 2015, 3, e00463-15. [CrossRef]

41. Egelkamp, R.; Schneider, D.; Hertel, R.; Daniel, R. Nitrile-Degrading Bacteria Isolated from Compost. Front. Environ. Sci. 2017, 5, 56. [CrossRef]

42. Blom, J.; Kreis, J.; Spänig, S.; Juhre, T.; Bertelli, C.; Ernst, C.; Goesmann, A. EDGAR 2.0: An enhanced software platform for comparative gene content analyses. Nucleic Acids Res. 2016, 44, W22-W28. [CrossRef] [PubMed]

43. Mitić, N.; Miraula, M.; Selleck, C.; Hadler, K.S.; Uribe, E.; Pedroso, M.M.; Schenk, G. Catalytic Mechanisms of Metallohydrolases Containing Two Metal Ions. Adv. Protein Chem. Struct. Biol. 2014, 97, 49-81. [PubMed]

44. Jensen, S.O.; Reeves, P.R. Domain organisation in phosphomannose isomerases (types I and II). Biochim. Biophys. Acta 1998, 1382, 5-7. [CrossRef] 
45. Barry, S.M.; Challis, G.L. Recent advances in siderophore biosynthesis. Curr. Opin. Chem. Biol. 2009, 13, 205-215. [CrossRef] [PubMed]

46. Matelska, D.; Steczkiewicz, K.; Ginalski, K. Comprehensive classification of the PIN domain-like superfamily. Nucleic Acids Res. 2017, 45, 6995-7020. [CrossRef] [PubMed]

47. Troxell, B.; Hassan, H.M. Transcriptional regulation by Ferric Uptake Regulator (Fur) in pathogenic bacteria. Front. Cell. Infect. Microbiol. 2013, 3, 59. [CrossRef] [PubMed]

48. Johnson, C.M.; Grossman, A.D. Integrative and Conjugative Elements (ICEs): What They Do and How They Work. Annu. Rev. Genet. 2015, 49, 577-601. [CrossRef] [PubMed]

49. Wozniak, R.A.; Waldor, M.K. A toxin-antitoxin system promotes the maintenance of an integrative conjugative element. PLoS Genet. 2009, 5, e1000439. [CrossRef]

50. Bateman, A.; Martin, M.J.; O’Donovan, C.; Magrane, M.; Apweiler, R.; Alpi, E.; Antunes, R.; Arganiska, J.; Bely, B.; Bingley, M.; et al. UniProt: A hub for protein information. Nucleic Acids Res. 2015, 43, D204-D212.

51. Hunter, S.; Apweiler, R.; Attwood, T.K.; Bairoch, A.; Bateman, A.; Binns, D.; Bork, P.; Das, U.; Daugherty, L.; Duquenne, L.; et al. InterPro: the integrative protein signature database. Nucleic Acids Res. 2009, 37, D211-D215. [CrossRef] [PubMed]

52. Lescot, M.; Hingamp, P.; Kojima, K.K.; Villar, E.; Romac, S.; Veluchamy, A.; Boccara, M.; Jaillon, O.; Iudicone, D.; Bowler, C.; et al. Reverse transcriptase genes are highly abundant and transcriptionally active in marine plankton assemblages. ISME J. 2016, 10, 1134-1146. [CrossRef] [PubMed]

53. Caparrós-Martín, J.A.; McCarthy-Suárez, I.; Culiáñez-Macià, F.A. HAD hydrolase function unveiled by substrate screening: enzymatic characterization of Arabidopsis thaliana subclass I phosphosugar phosphatase AtSgpp. Planta 2013, 237, 943-954. [CrossRef] [PubMed]

54. Favrot, L.; Blanchard, J.S.; Vergnolle, O. Bacterial GCN5-Related N-Acetyltransferases: From Resistance to Regulation. Biochemistry 2016, 55, 989-1002. [CrossRef] [PubMed]

55. Loper, J.E.; Hassan, K.A.; Mavrodi, D.V.; Davis, E.W.; Lim, C.K.; Shaffer, B.T.; Elbourne, L.D.; Stockwell, V.O.; Hartney, S.L.; Breakwell, K.; et al. Comparative genomics of plant-associated pseudomonas spp.: Insights into diversity and inheritance of traits involved in multitrophic interactions. PLoS Genet. 2012, 8, e1002784. [CrossRef] [PubMed]

56. Van Der Voort, M.; Meijer, H.J.G.; Schmidt, Y.; Watrous, J.; Dekkers, E.; Mendes, R.; Dorrestein, P.C.; Gross, H.; Raaijmakers, J.M. Genome mining and metabolic profiling of the rhizosphere bacterium Pseudomonas sp. SH-C52 for antimicrobial compounds. Front. Microbiol. 2015, 6, 693. [CrossRef] [PubMed]

57. Cimermancic, P.; Medema, M.H.; Claesen, J.; Kurita, K.; Wieland Brown, L.C.; Mavrommatis, K.; Pati, A.; Godfrey, P.A.; Koehrsen, M.; Clardy, J.; et al. Insights into secondary metabolism from a global analysis of prokaryotic biosynthetic gene clusters. Cell 2014, 158, 412-421. [CrossRef] [PubMed]

58. Chowdhury, S.P.; Hartmann, A.; Gao, X.W.; Borriss, R. Biocontrol mechanism by root-associated Bacillus amyloliquefaciens FZB42-A review. Front. Microbiol. 2015, 6, 780. [CrossRef]

59. Doornbos, R.F.; Van Loon, L.C.; Bakker, P.A. Impact of root exudates and plant defense signaling on bacterial communities in the rhizosphere. A review. Agron. Sustain. Dev. 2012, 32, 227-243. [CrossRef]

60. Venturi, V.; Keel, C. Signaling in the Rhizosphere. Trends Plant Sci. 2016, 21, 187-198. [CrossRef]

61. Yi, H.S.; Ahn, Y.R.; Song, G.C.; Ghim, S.Y.; Lee, S.; Lee, G.; Ryu, C.M. Impact of a bacterial volatile 2,3-butanediol on Bacillus subtilis rhizosphere robustness. Front. Microbiol. 2016, 7, 993. [CrossRef] [PubMed]

62. Duan, J.; Jiang, W.; Cheng, Z.; Heikkila, J.J.; Glick, B.R. The complete genome sequence of the plant growth-promoting bacterium Pseudomonas sp. UW4. PLoS ONE 2013, 8, e58640. [CrossRef] [PubMed]

63. Vitreschak, A.G. Regulation of riboflavin biosynthesis and transport genes in bacteria by transcriptional and translational attenuation. Nucleic Acids Res. 2002, 30, 3141-3151. [CrossRef] [PubMed]

64. Dakora, F.D.; Matiru, V.N.; Kanu, A.S. Rhizosphere ecology of lumichrome and riboflavin, two bacterial signal molecules eliciting developmental changes in plants. Front. Plant Sci. 2015, 6, 700. [CrossRef] [PubMed]

65. Deng, W.; Marshall, N.C.; Rowland, J.L.; McCoy, J.M.; Worrall, L.J.; Santos, A.S.; Strynadka, N.C.; Finlay, B.B. Assembly, structure, function and regulation of type III secretion systems. Nat. Rev. Microbiol. 2017, 15, 323-337. [CrossRef] [PubMed]

66. Preston, G.M.; Bertrand, N.; Rainey, P.B. Type III secretion in plant growth-promoting Pseudomonas fluorescens SBW25. Mol. Microbiol. 2001, 41, 999-1014. [CrossRef] 
67. Alori, E.T.; Glick, B.R.; Babalola, O.O. Microbial phosphorus solubilization and its potential for use in sustainable agriculture. Front. Microbiol. 2017, 8, 971. [CrossRef]

68. Rodríguez, H.; Fraga, R.; Gonzalez, T.; Bashan, Y. Genetics of phosphate solubilization and its potential applications for improving plant growth-promoting bacteria. Plant Soil 2007, 287, 15-21. [CrossRef]

69. Molina-Henares, M.A.; García-Salamanca, A.; Molina-Henares, A.J.; De La Torre, J.; Herrera, M.C.; Ramos, J.L.; Duque, E. Functional analysis of aromatic biosynthetic pathways in Pseudomonas putida KT2440. Microb. Biotechnol. 2009, 2, 91-100. [CrossRef]

70. Lamb, A.L. Breaking a pathogen's iron will: Inhibiting siderophore production as an antimicrobial strategy. Biochim. Biophys. Acta (BBA) Proteins Proteom. 2015, 1854, 1054-1070. [CrossRef]

71. Serino, L.; Reimmann, C.; Visca, P.; Beyeler, M.; Chiesa, V.D.; Haas, D. Biosynthesis of pyochelin and dihydroaeruginoic acid requires the iron-regulated pchDCBA operon in Pseudomonas aeruginosa. J. Bacteriol. 1997, 179, 248-257. [CrossRef] [PubMed]

72. Young, I.G.; Gibson, F. Regulation of the enzymes involved in the biosynthesis of 2,3-dihydroxybenzoic acid in Aerobacter aerogenes and Escherichia coli. BBA Gen. Subj. 1969, 177, 401-411. [CrossRef]

73. Pupin, M.; Esmaeel, Q.; Flissi, A.; Dufresne, Y.; Jacques, P.; Leclère, V. Norine: A powerful resource for novel nonribosomal peptide discovery. Synth. Syst. Biotechnol. 2016, 1, 89-94. [CrossRef] [PubMed]

74. Mann, E.E.; Wozniak, D.J. Pseudomonas biofilm matrix composition and niche biology. FEMS Microbiol. Rev. 2012, 36, 893-916. [CrossRef]

75. Malanovic, N.; Lohner, K. Gram-positive bacterial cell envelopes: The impact on the activity of antimicrobial peptides. Biochim. Biophys. Acta Biomembr. 2016, 1858, 936-946. [CrossRef]

76. Mellgren, E.M.; Kloek, A.P.; Kunkel, B.N. Mqo, a tricarboxylic acid cycle enzyme, is required for virulence of Pseudomonas syringae pv. tomato strain DC3000 on Arabidopsis thaliana. J. Bacteriol. 2009, 191, 3132-3141. [CrossRef]

77. Neupane, S.; Finlay, R.D.; Alström, S.; Elfstrand, M.; Högberg, N. Transcriptional responses of the bacterial antagonist Serratia plymuthica to the fungal phytopathogen Rhizoctonia solani. Environ. Microbiol. Rep. 2015, 7, 123-127. [CrossRef]

78. Perazzolli, M.; Herrero, N.; Sterck, L.; Lenzi, L.; Pellegrini, A.; Puopolo, G.; Van de Peer, Y.; Pertot, I. Transcriptomic responses of a simplified soil microcosm to a plant pathogen and its biocontrol agent reveal a complex reaction to harsh habitat. BMC Genom. 2016, 17, 838. doi:10.1186/s12864-016-3174-4. [CrossRef]

79. Achouak, W.; Conrod, S.; Cohen, V.; Heulin, T. Phenotypic Variation of Pseudomonas brassicacearum as a Plant Root-Colonization Strategy. Mol. Plant-Microbe Interact. 2004, 17, 872-879. [CrossRef]

80. Van Den Broek, D.; Bloemberg, G.V.; Lugtenberg, B. The role of phenotypic variation in rhizosphere Pseudomonas bacteria. Environ. Microbiol. 2005, 7, 1686-1697. [CrossRef]

81. Sajitha, K.L.; Dev, S.A. Quantification of antifungal lipopeptide gene expression levels in Bacillus subtilis B1 during antagonism against sapstain fungus on rubberwood. Biol. Control 2016, 96, 78-85. [CrossRef]

82. Lu, X.; Zhou, D.; Chen, X.; Zhang, J.; Huang, H.; Wei, L. Isolation and characterization of Bacillus altitudinis JSCX-1 as a new potential biocontrol agent against Phytophthora sojae in soybean [Glycine max (L.) Merr.]. Plant Soil 2017, 416, 53-66. [CrossRef]

83. Blauth de Lima, F.; da Silva Ribeiro, R.T.; Félix, C.; Osório, N.; Alves, A.; Esteves, A.C.; Vitorino, R.; Domingues, P. Trichoderma harzianum T1A constitutively secretes proteins involved in the biological control of Guignardia citricarpa. Biol. Control 2017, 106, 99-109. [CrossRef]

84. Puehringer, S.; Metlitzky, M.; Schwarzenbacher, R. The pyrroloquinoline quinone biosynthesis pathway revisited: A structural approach. BMC Biochem. 2008, 9, 8. [CrossRef] [PubMed]

85. Han, S.H.; Kim, C.H.; Lee, J.H.; Park, J.Y.; Cho, S.M.; Park, S.K.; Kim, K.Y.; Krishnan, H.B.; Kim, Y.C. Inactivation of pqq genes of Enterobacter intermedium $60-2 \mathrm{G}$ reduces antifungal activity and induction of systemic resistance. FEMS Microbiol. Lett. 2008, 282, 140-146. [CrossRef] [PubMed]

86. Hernández, V.M.; Girard, L.; Hernández-Lucas, I.; Vázquez, A.; Ortíz-Ortíz, C.; Díaz, R.; Dunn, M.F. Genetic and biochemical characterization of arginine biosynthesis in Sinorhizobium meliloti 1021. Microbiology 2015, 161, 1671-1682. [CrossRef] [PubMed]

87. Zúñiga, M.; Pérez, G.; González-Candelas, F. Evolution of arginine deiminase (ADI) pathway genes. Mol. Phylogenetics Evol. 2002, 25, 429-444. [CrossRef]

88. Pitcher, R.S.; Watmough, N.J. The bacterial cytochrome cbb3 oxidases. Biochim. Biophys. Acta 2004, 1655, 388-399. [CrossRef] 
89. Hilker, R.; Stadermann, K.B.; Schwengers, O.; Anisiforov, E.; Jaenicke, S.; Weisshaar, B.; Zimmermann, T.; Goesmann, A. ReadXplorer 2-Detailed read mapping analysis and visualization from one single source. Bioinformatics 2016, 32, 3702-3708. [CrossRef]

90. Krishna, S.S.; Aravind, L.; Bakolitsa, C.; Caruthers, J.; Carlton, D.; Miller, M.D.; Abdubek, P.; Astakhova, T.; Axelrod, H.L.; Chiu, H.J.; et al. The structure of SSO2064, the first representative of Pfam family PF01796, reveals a novel two-domain zinc-ribbon OB-fold architecture with a potential acyl-CoA-binding role. Acta Crystallogr. Sect. F Struct. Biol. Crystall. Commun. 2010, 66, 1160-1166. [CrossRef]

91. Zhang, L.; Xu, Q.; Zhan, S.; Li, Y.; Lin, H.; Sun, S.; Sha, L.; Hu, K.; Guan, X.; Shen, Y. A new $\mathrm{NAD}(\mathrm{H})$-dependent meso-2,3-butanediol dehydrogenase from an industrially potential strain Serratia marcescens H30. Appl. Microbiol. Biotechnol. 2014, 98, 1175-1184. [CrossRef] [PubMed]

92. Ryu, C.M.; Farag, M.A.; Hu, C.H.; Reddy, M.S.; Wei, H.X.; Pare, P.W.; Kloepper, J.W. Bacterial volatiles promote growth in Arabidopsis. Proc. Natl. Acad. Sci. USA 2003, 100, 4927-4932. [CrossRef] [PubMed]

93. Gutiérrez-Preciado, A.; Torres, A.G.; Merino, E.; Bonomi, H.R.; Goldbaum, F.A.; García-Angulo, V.A. Extensive identification of bacterial riboflavin transporters and their distribution across bacterial species. PLoS ONE 2015, 10, e0126124. [CrossRef] [PubMed]

94. García-Angulo, V.A. Overlapping riboflavin supply pathways in bacteria. Crit. Rev. Microbiol. 2017, 43, 196-209. [CrossRef] [PubMed]

95. Li, H.; Durbin, R. Fast and accurate long-read alignment with Burrows-Wheeler transform. Bioinformatics 2010, 26, 589-595. [CrossRef]

96. Koboldt, D.C.; Zhang, Q.; Larson, D.E.; Shen, D.; McLellan, M.D.; Lin, L.; Miller, C.A.; Mardis, E.R.; Ding, L.; Wilson, R.K. VarScan 2: Somatic mutation and copy number alteration discovery in cancer by exome sequencing. Genome Res. 2012, 22, 568-576. [CrossRef]

97. Tatusova, T.; Dicuccio, M.; Badretdin, A.; Chetvernin, V.; Nawrocki, E.P.; Zaslavsky, L.; Lomsadze, A.; Pruitt, K.D.; Borodovsky, M.; Ostell, J. NCBI prokaryotic genome annotation pipeline. Nucleic Acids Res. 2016, 44, 6614-6624. [CrossRef]

98. van Heel, A.J.; de Jong, A.; Montalbán-López, M.; Kok, J.; Kuipers, O.P. BAGEL3: Automated identification of genes encoding bacteriocins and (non-)bactericidal posttranslationally modified peptides. Nucleic Acids Res. 2013, 41, W448-W453. [CrossRef]

99. Weber, T.; Blin, K.; Duddela, S.; Krug, D.; Kim, H.U.; Bruccoleri, R.; Lee, S.Y.; Fischbach, M.A.; Müller, R.; Wohlleben, W.; et al. AntiSMASH 3.0-A comprehensive resource for the genome mining of biosynthetic gene clusters. Nucleic Acids Res. 2015, 43, W237-W243. [CrossRef]

100. Flissi, A.; Dufresne, Y.; Michalik, J.; Tonon, L.; Janot, S.; Noé, L.; Jacques, P.; Leclére, V.; Pupin, M. Norine, the knowledgebase dedicated to non-ribosomal peptides, is now open to crowdsourcing. Nucleic Acids Res. 2016, 44, D1113-D1118. [CrossRef]

101. Sneath, P.H.A.; McGowan, V.; Skerman, V.B.D. Approved lists of bacterial names. Med. J. Aust. 1980, 2, 3-4.

102. Elomari, M.; Coroler, L.; Hoste, B.; Gillis, M.; Izard, D.; Leclerc, H. DNA Relatedness among Pseudomonas Strains Isolated from Natural Mineral Waters and Proposal of Pseudomonas veronii sp. nov. Int. J. Syst. Bacteriol. 2009, 46, 1138-1144. [CrossRef] [PubMed]

103. Behrendt, U.; Ulrich, A.; Schumann, P. Fluorescent pseudomonads associated with the phyllosphere of grasses; Pseudomonas trivialis sp. nov., Pseudomonas poae sp. nov. and Pseudomonas congelans sp. nov. Int. J. Syst. Evol. Microbiol. 2003, 53, 1461-1469. [CrossRef] [PubMed]

104. Euzéby, J. Validation of publication of new names and new combinations previously effectively published outside the USEM. Int. J. Syst. Evol. Microbiol. 2004, 54, 631-632.

105. Edgar, R.C. MUSCLE: Multiple sequence alignment with high accuracy and high throughput. Nucleic Acids Res. 2004, 32, 1792-1797. [CrossRef] [PubMed]

106. He, Z.; Zhang, H.; Gao, S.; Lercher, M.J.; Chen, W.H.; Hu, S. Evolview v2: An online visualization and management tool for customized and annotated phylogenetic trees. Nucleic Acids Res. 2016, 44, W236-W241. [CrossRef] [PubMed]

107. Meier-Kolthoff, J.P.; Auch, A.F.; Klenk, H.P.; Göker, M. Genome sequence-based species delimitation with confidence intervals and improved distance functions. BMC Bioinform. 2013, 14, 60. [CrossRef]

108. Grosch, R.; Schneider, J.H.; Kofoet, A. Characterisation of Rhizoctonia solani anastomosis groups causing bottom rot in field-grown lettuce in Germany. Eur. J. Plant Pathol. 2004, 110, 53-62. [CrossRef] 
109. Pátek, M.; Holátko, J.; Busche, T.; Kalinowski, J.; Nešvera, J. Corynebacterium glutamicum promoters: A practical approach. Microb. Biotechnol. 2013, 6, 103-117. [CrossRef]

110. Haas, B.J.; Chin, M.; Nusbaum, C.; Birren, B.W.; Livny, J. How deep is deep enough for RNA-Seq profiling of bacterial transcriptomes? BMC Genom. 2012, 13, 734. [CrossRef]

111. Bushnell, B. BBMap: A Fast, Accurate, Splice-Aware AlignerLawrence Berkeley National Lab.(LBNL), Berkeley, CA (United States). 2014. https://jgi.doe.gov/wp-content/uploads/2013/11/BB_User-Meeting2014-poster-FINAL.pdf (accessed on 7 August 2019).

112. Love, M.I.; Huber, W.; Anders, S. Moderated estimation of fold change and dispersion for RNA-seq data with DESeq2. Genome Biol. 2014, 15, 550. [CrossRef] [PubMed]

2019 by the authors. Licensee MDPI, Basel, Switzerland. This article is an open access article distributed under the terms and conditions of the Creative Commons Attribution (CC BY) license (http://creativecommons.org/licenses/by/4.0/). 